\title{
Communication \\ The Source Term of the Non-Equilibrium Statistical Operator
}

\author{
Gerd Röpke ${ }^{1,2}$ \\ 1 Institute of Physics, University of Rostock, D-18051 Rostock, Germany; gerd.roepke@uni-rostock.de \\ 2 Department of Theoretical Nuclear Physics, National Research Nuclear University (MEPhI), \\ 115409 Moscow, Russia
}

Received: 8 May 2019; Accepted: 4 June 2019; Published: 6 June 2019

\begin{abstract}
The method of Zubarev allows one to construct a statistical operator for the nonequilibrium. The von Neumann equation is modified introducing a source term that is considered as an infinitesimal small correction. This approach provides us with a very general and unified treatment of nonequilibrium processes. Considering as an example the electrical conductivity, we discuss the modification of the von Neumann equation to describe a stationary nonequilibrium process. The Zubarev approach has to be generalized to open quantum systems. The interaction of the system with the irrelevant degrees of freedom of the bath is globally described by the von Neumann equation with a finite source term. This is interpreted as a relaxation process to an appropriate relevant statistical operator. As an alternative, a quantum master equation can be worked out where the coupling to the bath is described by a dissipator. The production of entropy is analyzed.
\end{abstract}

Keywords: irreversibility; entropy; linear response theory; electrical conductivity

\section{Electrical Conductivity-Phenomenology}

Transport processes. The method of the nonequilibrium statistical operator (NSO) invented by D. N. Zubarev [1] is an important step working out a general approach to the statistical mechanics of nonequilibrium processes. It covers different fields of nonequilibrium theory, in particular the thermodynamics of irreversible processes, kinetic theory, linear response theory, open quantum systems, quantum master equations, and hydrodynamics; see [2,3]. The method of NSO provides us with a consistent and coherent approach to nonequilibrium statistics. However, it gives also a view of the sensible points, deriving equations of evolution for irreversible phenomena (e.g., transport processes and reaction rates) from the reversible basic equations of motion such as Hamilton equations, Maxwell theory, and quantum field theory.

Let us start with a simple example for a transport process, friction. Friction transforms mechanical work into heat. It is one of the fundamental processes that are considered to introduce irreversibility and the production of entropy according to the second law of thermodynamics.

A particular case is electrical conductivity. We consider a system containing two species of charged particles, the ions (charge $Z_{i} e$ ) and the electrons (charge $-e$ ), for instance a hydrogen plasma consisting of electrons and protons, or a piece of copper as a system of ions, fixed on lattice sites, and quasi-free electrons. The system is assumed to be charge neutral, so that the densities are related as $Z_{i} n_{i}=n_{e}$, where $n_{c}=\left\langle\mathrm{N}_{c}\right\rangle / \Omega_{0}$ is the average of the particle number $\mathrm{N}_{c}$ per volume $\Omega_{0}$. Without loss of generality, we assume in this work $Z_{i}=1$. Under the influence of a constant external electrical field $E_{\text {ext }}$, an electrical current with density $j$ is induced. As an empirical fact, below a critical value of $\left|E_{\text {ext }}\right|$, the current is proportional to the field. For isotropic systems, we have:

$$
j=\sigma E_{\mathrm{ext}}
$$


with the transport coefficient $\sigma$ being the electrical conductivity. Note that the electrical conductivity is defined with the screened, intrinsic electrical field, $j=\sigma E_{\text {int. }}$. In the case considered here, the intrinsic electrical field $\boldsymbol{E}_{\text {int }}$ coincides with the external field.

To have an irreversible process, the mechanical work $\Omega_{0} j \cdot E_{\text {ext }} \mathrm{d} t$ must be transformed to heat $Q$,

$$
\frac{1}{\Omega_{0}} \frac{\delta Q}{\mathrm{~d} t}=j \cdot \boldsymbol{E}_{\mathrm{ext}}=\frac{1}{\sigma} j^{2},
$$

if no other forms of work are performed by the system. The increase of heat is related to the production of entropy, $\mathrm{d} S=\delta Q / T_{\text {eq }}$, if the system remains near thermodynamic equilibrium with temperature $T_{\text {eq }}$.

We consider a quasi-stationary situation where the electrical field, in general $\boldsymbol{E}_{\text {ext }}(\boldsymbol{r}, t)$, and the current density, in general $j(r, t)$, are constant with respect to time. Furthermore, we consider the homogeneous case where both quantities are not depending on position. In addition, the densities $n_{c}$ of charged particles and the temperature $T$ are considered as constant, also not depending on position. We denote this as the external conditions that we demand from the experiment.

Open systems. These quasi-stationary, homogeneous conditions with constant electrical current density, particle number density, and temperature are only possible for open systems. We characterize them by the finite volume, here a cylinder $\Omega_{R}^{h}$ with the axis parallel to $j$ taken as the $z$ axis, between $z=0$ and $z=h$, and with radius $R$. The electrical field $E_{\text {ext }}=E_{\text {ext }} e_{z}$ is also directed along the $z$ axis. To sustain the constant electrical current, particles must be introduced through the surface at $z=h$ (with high potential energy $V_{\text {ext }}(h)$ ) and extracted through the opposite surface at $z=0$ (with lower potential energy $V_{\text {ext }}(0)$ ). The difference of the potential energy of an electron is $V_{\text {ext }}(h)-V_{\text {ext }}(0)=e h E_{\text {ext }}$. Without loss of generality, we omit the current of ions.

The particles injected in the open system at high potential energy gain kinetic energy according to the conservation law in mechanics, before they leave the open system at low potential energy. However, because of the demand of homogeneity in space, the electron current that leaves the system transports the same amount of kinetic energy as the incoming electron current. According to the first law of thermodynamics, mechanical work is transformed to thermal energy. Heat is produced at the rate per volume (power density) according to Equation (2).

More precisely, to stay in quasi-equilibrium, this amount of energy must be extracted from the system to a bath. Instead of an isolated, closed system as frequently considered in physics, described by well-defined dynamical degrees of freedom, we have to consider an open system for the stationary transport process. The contact with the surroundings (the "bath", or additional degrees of freedom) is necessary not only to sustain the current of electrons, but also for the export of heat to sustain a constant temperature. We assume for the bath thermodynamic equilibrium at the external (bath) temperature $T_{\text {ext }}$. Below, in Section 8, we consider it as a local property $T_{\text {ext }}(r, t)$. In the case that the open system is in contact with a material bath, we have thermal conductivity, e.g., by phonon transport. In a vacuum, the transport of energy is performed by radiation. In particular, the bremsstrahlung may be the primary process to transform mechanical energy into the energy of radiation. Temperature and heat for radiation are defined after absorption by a hohlraum, where the Planck spectrum of radiation is established in equilibrium.

The concept of heat is introduced as a process to export energy to a bath. According to the second law of thermodynamics, the density of entropy production results as:

$$
\frac{1}{\Omega_{R}^{h}} \frac{\mathrm{d} S}{\mathrm{~d} t}=\frac{1}{T_{\mathrm{ext}} \sigma} j^{2} .
$$

The second law of thermodynamics implies $\sigma>0$ (the flow of a river is never up-hill).

As a more general case, periodic dependence in time and space can be considered, and the optical conductivity or AC conductivity $\sigma(\boldsymbol{q}, \omega)$ depending on the wave vector $\boldsymbol{q}$ and the frequency $\omega$ is introduced. Because of linearity, a general dependence on space and time, such as the switch-on situation where the field $E_{\text {ext }}(t)$ is proportional to the step function, is treated via Fourier decomposition 
and superposition of the solution for the components. This general case can also be treated with our approach [4-6], but is not considered in this work, where we focus on the coupling to a bath.

\section{Electrical Conductivity-Microscopic Approach}

Microscopic model. We construct a microscopic approach to friction and electrical conductivity, which are typical irreversible processes. This means we start from the well-known equations of motion for the particles and fields according to quantum mechanics and quantum electrodynamics. These equations, as well as their classical limits (Newton's or Maxwell's equations) describe reversible motion. We consider a simple microscopic model: Electrons move in a system of heavy ions (at positions $\boldsymbol{R}_{i}$ ) under the influence of an external field $\boldsymbol{E}_{\text {ext }}$. Within the Lorentz model, the electron-electron interaction is replaced by a mean field to ensure charge neutrality. The given electron-ion interaction $V_{\mathrm{ei}}(\boldsymbol{r})$ defines the Hamiltonian that characterizes the model system:

$$
\mathrm{H}_{\mathrm{S}}=\sum_{p} E_{p} \mathrm{a}_{p}^{\dagger} \mathrm{a}_{p}+\sum_{p, \boldsymbol{q}} V(\boldsymbol{q}) \mathrm{a}_{p+\boldsymbol{q}}^{\dagger} \mathrm{a}_{p}
$$

where $p=\{\boldsymbol{p}, \hat{\sigma}\}$ is the single-electron state with wave vector $p$. The spin $\hat{\sigma}$ is treated implicitly. $E_{p}=\hbar^{2} p^{2} / 2 m_{e}$ is the kinetic energy, and $V(\boldsymbol{q})=\Omega_{0}^{-1} \sum_{i}^{N_{i}} \int \mathrm{d}^{3} r e^{\mathrm{i} \boldsymbol{q} \cdot \boldsymbol{r}} V_{\mathrm{ei}}\left(\boldsymbol{r}-\boldsymbol{R}_{i}\right)$ is the Fourier transform of the interaction with all ions at positions $\boldsymbol{R}_{i}$.

In addition, the influence of the external field $\boldsymbol{E}_{\text {ext }}(t)$ (which in general may depend on time and space) is described by the contribution (electron charge $-e$ ):

$$
\mathrm{H}_{\mathrm{F}}^{t}=e \mathbf{R} \cdot \boldsymbol{E}_{\mathrm{ext}}(t)
$$

with the electron position operator $\mathbf{R}=\sum_{i}^{N_{e}} \boldsymbol{r}_{i}$. This is the sum of the potential energies of all electrons in the system. The total Hamiltonian $\mathrm{H}^{t}=\mathrm{H}_{\mathrm{S}}+\mathrm{H}_{\mathrm{F}}^{t}$ determines the motion of the electrons in the microscopic approach, i.e., the dynamics of the system.

Without electron-ion interaction, the solution of the equation of motion for the electrons is simple. The momentum $\hbar \mathbf{p}=m_{e} \dot{\mathbf{r}}$ of each electron is changed with time according to $\hbar \dot{\mathbf{p}}_{i}=-e \boldsymbol{E}_{\text {ext }}$. We consider the cylindrical volume $\Omega_{R}^{\mathrm{d} z}$ with height $h=\mathrm{d} z$. An electron incoming at $\mathrm{d} z$ with momentum $\hbar p, p_{z}<0$ and leaving the volume at $z=0$ after $\mathrm{d} t=\left(-m_{e} / \hbar p_{z}\right) \mathrm{d} z$ will have the $z$ component of momentum $\hbar p_{z}+e E_{\text {ext }}\left(m_{e} / \hbar p_{z}\right) \mathrm{d} z$. This corresponds to an increase of kinetic energy by:

$$
\mathrm{d} E_{\text {kin }}=\frac{1}{2 m_{e}} 2 e E_{\text {ext }} m_{e} \mathrm{~d} z+\mathcal{O}\left(\mathrm{d} z^{2}\right)
$$

equal to the loss $e E_{\text {ext }} \mathrm{d} z$ of potential energy, as given by Equation (5). The acceleration of all incoming electrons to outgoing electrons makes the average momentum and the corresponding electrical current dependent on the position, which is in contradiction with the requested homogeneity.

The electron-ion interaction, Equation (4), destroys the increase of the average momentum in the $z$ direction by scattering, which changes the direction of the momentum. In the adiabatic limit $M_{i} / m_{e} \rightarrow \infty$, we have elastic scattering. The average momentum of the electron system gained by the electrical field is transferred to the ion system (and is compensated by the ion system because of charge neutrality). The loss of average momentum of the electron subsystem defines the stationary current and the conductivity, as calculated below for a given $V_{\mathrm{ei}}(\boldsymbol{q})$. Nevertheless, the Hamiltonian $\mathrm{H}^{t}=\mathrm{H}_{\mathrm{S}}+\mathrm{H}_{\mathrm{F}}^{t}$ is not sufficient to describe the process of stationary conductivity because it does not describe the contact with the bath, in particular how the electrons enter and leave the open system and how energy is dissipated.

Contact with the bath. The conservation of particle number leads to the balance equation for the particle current and is described by the incoming and outgoing currents of the open system $\Omega_{0}$. It is assumed that the process of injection and extraction of electrons to sustain the current in the open system is not relevant for the calculation of the conductivity. We can circumvent this problem 
considering a larger system $\bar{\Omega}$ where the particle number is conserved. This may be a circuit consisting of a capacitor, an inductor, and a resistor, driven by periodic external electromagnetic fields, so that a quasi-stationary state with forced oscillations with $\omega_{0}$ is obtained. Examples are absorbed radiation by an antenna or the second circuit of a transformer.

Let us consider the induction of electrical fields by the change of magnetic field with time, expressed via the vector potential $A_{\mathrm{ext}}(\boldsymbol{r}, t)$ (Coulomb gauge), so that:

$$
E_{\text {ext }}(r, t)=-\frac{\partial}{c \partial t} A_{\text {ext }}(r, t) .
$$

The coupling to the external field $\boldsymbol{A}_{\text {ext }}(\boldsymbol{r}, t)$ is given by the expression $(e \hbar / c) \mathbf{p} \cdot \boldsymbol{A}_{\text {ext }}$, so that the average absorbed power density is $-j \cdot \dot{A}_{\text {ext }} / c=j \cdot E_{\text {ext }}$. The expression:

$$
\mathrm{H}_{\mathrm{F}}^{t}=\frac{e \hbar}{c} \sum_{p} p \cdot A_{\mathrm{ext}}(t) \mathrm{a}_{p}^{\dagger} \mathrm{a}_{p}
$$

has the advantage that it is expressed in second quantization with respect to momentum states, and the average current density:

$$
j=\frac{-e \hbar}{m_{e} \Omega_{0}} \operatorname{Tr}\left\{\rho \sum_{i}^{N_{e}} \mathbf{p}_{i}\right\}=\frac{-e \hbar}{m_{e} \Omega_{0}} \sum_{p} p\left\langle\mathrm{a}_{p}^{\dagger} \mathrm{a}_{p}\right\rangle
$$

does not depend on position as demanded for the homogeneous situation. Now, we can relax the problem of particle exchange with the bath because there is particle conservation for the closed circuit.

However, we cannot eliminate the bath with respect to the absorption of the heat, which is produced by the electrical current flowing across the resistor. The motion of the electrons in the external field gives an average increase of kinetic energy. The transfer of electrical energy to mechanical energy per volume and time is given by $\boldsymbol{j} \cdot \boldsymbol{E}_{\mathrm{ext}}$. However, the external conditions are given so that not only the current density, but also the temperature is constant. This is in conflict with energy conservation. For the condition that in the stationary case, the electron system is homogeneous, i.e., the averages are not varying with position, the export of energy cannot be done by the electron system across the surface of the system, but needs other mechanisms, denoted as coupling to a thermal bath.

A standard device is the export of energy by coupling to a material thermal bath consisting of matter, which is characterized by the average kinetic energy according to a temperature $T_{\text {ext }}$. The coupling is mediated by collisions between the ions and can be expressed by phonons. As a characteristic of the bath, back-reaction is excluded, and coherence and correlations are destructed. This refers, in particular, to the phase of the phonons. The phase is defined in a coherent state, also in the classical description of the electron-ion interaction, but not in quantum-statistical thermal equilibrium, which is described by occupation numbers of the phonon states.

More general, without the need for a material bath, is the radiation field, which is always present. The emission of photons is a well-known effect for the export of energy, but this is not the solution of the problem of irreversibility. A photon has a definite energy, and a radio wave a definite phase. Neither are thermalized. Only the Planck hohlraum radiation is thermalized and is characterized by the equilibrium temperature $T_{\text {eq }}$. This black-body radiation is given by the Bose occupation numbers for the single-photon states:

$$
n_{\mathrm{B}}\left(\omega, T_{\mathrm{eq}}\right)=\frac{1}{e^{\hbar \omega / k_{\mathrm{B}} T_{\mathrm{eq}}-1}}, \quad \varrho\left(\omega, T_{\mathrm{eq}}\right)=\frac{\hbar \omega^{3}}{4 \pi^{3} c^{2}} n_{\mathrm{B}}\left(\omega, T_{\mathrm{eq}}\right)
$$

is the spectral radiance of blackbodies, i.e., the power emitted from the emitting surface, per unit projected area of emitting surface, per unit solid angle, per angular frequency unit. We use in this work the Planck hohlraum radiation to define heat and $T_{\text {eq }}$. Other degrees of freedom, in particular 
the kinetic energy of the plasma constituents, may be in thermal equilibrium with the black-body radiation, which defines the bath. Then, the average energy per classical degree of freedom is $k_{\mathrm{B}} T_{\mathrm{eq}} / 2$.

Flow of energy. Coming back to the DC conductivity, we discuss the flow of energy. The stationary current with density $j$ induces a magnetic field, and with the constant electrical field $E_{\text {ext }}$, the Poynting vector is obtained, which describes the flow density of electromagnetic energy from outer space into the material. The energy current density of the electromagnetic field is given by the Poynting vector $S_{\text {ext }}=E_{\text {ext }} \times B_{\text {ext }}$. For the cylindrical configuration considered here, this is a radial vector perpendicular to the $z$ axis. For the current density $j$, at radius $R$, the value $B_{\text {ext }}=\pi R^{2} j / 2 \pi R$ results, so that the energy current of the electromagnetic field into the volume $\Omega_{R}^{h}=\pi R^{2} h$ comes out as the surface integral $\int \mathrm{d} O \cdot S_{\text {ext }}=j E_{\text {ext }} R / 2 \times 2 \pi R h=j E_{\text {ext }} \Omega_{R}^{h}$. This verifies the energy conservation: the power density $j E_{\text {ext }}$ absorbed by the electrons moving in the electrical field is imported via the electromagnetic energy density current $S_{\text {ext }}$. There, it is transformed into mechanical energy, according to the reversible Maxwell equations of motion for the electromagnetic fields. In the stationary case, the amount of imported power has to be exported.

We consider the energy export from the system by thermal conduction and thermal radiation. In both cases, the formation of a Planck spectrum with a definite temperature $T_{\text {ext }}$ and the corresponding black-body radiation describes the distribution of the energy with respect to the quantum states, and this energy can be addressed as heat. The export of energy is also possible in different forms, such as performing work by evaporating particles with high kinetic energy, phonons that are not thermalized, or emitting photons out of thermal equilibrium. Thermal conduction can be realized via the ion system by individual collisions with ions or collective excitations (phonons). The bottleneck is the transfer of kinetic energy from the light electrons, mass $m_{e}$, to the heavy ions, mass $M_{i}$, owing to collisions. Here, the ratio $m_{e} / M_{i}$ determines the transfer of energy. In the second case of photon emission, radiation transport does not need contact with a material bath, but the omnipresent vacuum.

Entropy production. In addition, the production of entropy is of interest, which is related to the production of heat. We introduced heat as a property of the electromagnetic field, the black-body radiation. The transfer from external field energy to mechanical energy gives no change in the entropy. Similarly, the export of energy out of thermal equilibrium is not connected with the entropy production. Only the transformation to heat gives an increase of entropy. The formation of a Planck spectrum and the corresponding black-body radiation can be addressed as heat. We need a discussion of the bath, as well as the coupling of the system to the bath. We focus on the Maxwell field as the bath, but many relations can also be discussed for a phonon bath.

Bremsstrahlung radiation. We consider in this work radiation transport. The free-free transitions of electrons moving under the influence of an interaction potential, here the ion potential, lead to the emission or absorption of the bremsstrahlung. The emission of radiation by a plasma is characterized by the emission coefficient $j(\omega)$, which gives the rate of radiation energy per unit volume, frequency $\omega$, and solid angle. For a system in thermal equilibrium, the emission coefficient is related to the absorption coefficient $\alpha(\omega)$ by Kirchhoff's law:

$$
j_{\mathrm{em}}(\omega)=\frac{\hbar \omega^{3}}{4 \pi^{3} c^{2}} \frac{1}{e^{\hbar \omega / k_{\mathrm{B}} T_{\mathrm{eq}}}-1} \alpha(\omega)
$$

where the spectral power density of blackbody radiation appears, and $c$ is the speed of light. This expression can be obtained from the Larmor formula for the radiated power of an accelerated single electron,

$$
P=-\frac{\mathrm{d} E}{\mathrm{~d} t}=\frac{e^{2}}{6 \pi \epsilon_{0} c^{3}}(\ddot{r})^{2}
$$


The thermally-averaged emission coefficient for a non-relativistic plasma considering free-free transitions without particle correlations reads according to Kramers [7]:

$$
j_{\mathrm{em}}(\omega)=\frac{e^{6} Z_{i}^{2} n_{e} n_{i}}{24 \pi^{3} \sqrt{6 \pi} \epsilon_{0}^{3} c^{3} m_{e}^{3 / 2}} \frac{e^{-\hbar \omega / k_{\mathrm{B}} T_{\mathrm{eq}}}}{\sqrt{k_{\mathrm{B}} T_{\mathrm{eq}}}} \bar{g}_{\mathrm{ff}}(\omega)
$$

with $\bar{g}_{\text {fff }}(\omega) \approx 1$. The Gaunt factor $\bar{g}_{\mathrm{ff}}(\omega)$ was introduced by Gaunt [8] in order to account for quantum-mechanical modifications; see also Section 6 below.

However, the bremsstrahlung radiation emitted during the electron-ion collision has to be transported across the plasma where self-absorption may occur. If self-absorption can be neglected, the plasma is optically thin, and the radiation can escape. If the plasma becomes optically thick, radiation propagates only a short distance before it will be absorbed. At frequencies $\omega$ below the plasma frequency, $\omega<\omega_{\mathrm{pl}}=\sqrt{e^{2} n_{e} / \epsilon_{0} m_{e}}$, radiation cannot freely propagate. In the optically-thick plasma, the upper limit for the emitted radiation is determined by the Planck Formula (10), see also Section 8. The low-frequency part of the radiation spectrum follows the Raleigh-Jeans law $\varrho\left(\omega, T_{\mathrm{eq}}\right) \approx \omega^{2} k_{\mathrm{B}} T_{\mathrm{eq}} / 4 \pi^{3} c^{2}$. It defines the temperature $T_{\mathrm{eq}}$ and, via the Planck formula, the heat deposited in the radiation field.

\section{Semiempirical Calculation of the Conductivity of the Adiabatic Lorentz Plasma}

Boltzmann equation. In the next step, after discussing microscopic properties of the system and the bath, we come back to the calculation of the conductivity of the adiabatic Lorentz plasma in the low-density limit. A semiempirical approach, the Boltzmann equation, considers the transfer of the ordered, directed motion of electrons to disordered motion owing to collisions. We consider the single-particle distribution function $f_{1}(p, t)$. Because of homogeneity in space, in the classical case, there is no dependence on position $r$. In the quantum case, we have the diagonal elements $f_{1}(p, t)=\left\langle\mathrm{a}_{p}^{\dagger} \mathrm{a}_{p}\right\rangle^{t}$ of the density matrix; the spin $\hat{\sigma}$ in $p=\{\boldsymbol{p}, \hat{\sigma}\}$ is not treated explicitly. The average is performed with the statistical operator $\rho(t)$. In the inhomogeneous case, the non-diagonal elements of the density matrix lead to the $r$ dependence of the Wigner function. In equilibrium, neglecting interaction in the low-density limit, the electron distribution is given by the ideal Fermi gas:

$$
f_{1}^{0}\left(E_{p}\right)=\frac{1}{e^{\beta\left(E_{p}-\mu_{e}\right)}+1}, \quad E_{p}=\frac{\hbar^{2} p^{2}}{2 m_{e}}, \quad \beta=\frac{1}{k_{\mathrm{B}} T_{\mathrm{eq}}} .
$$

The electron chemical potential $\mu_{e}$ is calculated from the electron density $n_{e}(t)=\Omega_{0}^{-1} \sum_{p} f_{1}(p, t)$. The electrical current density of electrons, charge $-e$,

$$
j(t)=\frac{-e \hbar}{m_{e} \Omega_{0}} \sum_{p} p f_{1}(p, t)
$$

is zero in equilibrium.

Owing to external fields and collisions among particles, the distribution function changes with time. According to Boltzmann [9], we have:

$$
\frac{\partial}{\partial t} f_{1}=\left(\frac{\partial}{\partial t} f_{1}\right)_{\mathrm{D}}+\left(\frac{\partial}{\partial t} f_{1}\right)_{\mathrm{St}}
$$

which becomes zero for the stationary state. The drift term contains the external force, with $v=$ $\hbar p / m_{e}$ following:

$$
\left(\frac{\partial}{\partial t} f_{1}\right)_{\mathrm{D}}=-\boldsymbol{v} \frac{\partial}{\partial r} f_{1}(\boldsymbol{p})-\boldsymbol{F}_{\mathrm{ext}} \frac{\partial}{\hbar \partial \boldsymbol{p}} f_{1}(\boldsymbol{p})=e \boldsymbol{E}_{\mathrm{ext}} \frac{\partial}{\hbar \partial \boldsymbol{p}} f_{1}(\boldsymbol{p})
$$


for the homogeneous case. Note that a mean-field term can be added to the external force. The internal interactions are contained in the collision term $\left(\partial f_{1} / \partial t\right)_{\mathrm{St}}$ for which, from the BBGKYhierarchy, an exact expression can be given containing the two-particle distribution function [10]. As an approximation, we assume a balance between gain and loss, $\left(\partial f_{1} / \partial t\right)_{S t}=G-L$. With some phenomenological considerations, we find in the quantum case the collision term as:

$$
\left(\frac{\partial}{\partial t} f_{1}\right)_{\mathrm{St}}=\int \frac{\mathrm{d}^{3} \boldsymbol{p}^{\prime} \Omega_{0}}{(2 \pi)^{3}}\left\{f_{1}\left(\boldsymbol{p}^{\prime}\right) w_{p \boldsymbol{p}^{\prime}}\left(1-f_{1}(\boldsymbol{p})\right)-f_{1}(\boldsymbol{p}) w_{\boldsymbol{p}^{\prime} \boldsymbol{p}}\left(1-f_{1}\left(\boldsymbol{p}^{\prime}\right)\right)\right\},
$$

where $w_{p p^{\prime}}$ is the transition rate from the momentum state $p^{\prime}$ to the state $p$. The quantum behavior of the collisions is taken into account via the Pauli blocking factors $\left(1-f_{1}(\boldsymbol{p})\right)$.

In the adiabatic limit, the interaction part of the Hamiltonian (4), matrix elements $H_{p^{\prime}}^{\prime}=V\left(\boldsymbol{p}^{\prime}-\boldsymbol{p}\right)$ describe elastic collisions. In Born approximation, the transition rate is given by Fermi's golden rule,

$$
w_{p^{\prime} p}=\frac{2 \pi}{\hbar}\left|H_{p^{\prime} p}^{\prime}\right|^{2} \delta\left(E_{p}-E_{p^{\prime}}\right)=w_{p p^{\prime}}
$$

Relaxation time method. To calculate the electrical conductivity, we make the ansatz that for small electrical fields, also the deviation of $f_{1}(\boldsymbol{p})$ from the equilibrium distribution $f_{1}^{0}\left(E_{p}\right)$ is small, and we assume a linear relation. The deviation from equilibrium is described by the function $\Phi(\boldsymbol{p})$ defined as (see also [4]):

$$
f_{1}(\boldsymbol{p})=f_{1}^{0}\left(E_{p}\right)-\Phi(\boldsymbol{p}) \frac{\mathrm{d} f_{1}^{0}\left(E_{p}\right)}{\mathrm{d} E_{p}} k_{\mathrm{B}} T_{\mathrm{eq}}=f_{1}^{0}\left(E_{p}\right)\left\{1+\Phi(\boldsymbol{p})\left(1-f_{1}^{0}\left(E_{p}\right)\right)\right\}
$$

For the equilibrium distribution $f_{1}^{0}\left(E_{p}\right)$, we have the detailed balance condition $w_{p p^{\prime}} f_{1}^{0}\left(E_{p^{\prime}}\right)(1-$ $\left.f_{1}^{0}\left(E_{p}\right)\right)=w_{p^{\prime}} f_{1}^{0}\left(E_{p}\right)\left(1-f_{1}^{0}\left(E_{p^{\prime}}\right)\right)$. Insertion of Equation (20) into the Boltzmann Equation (16) yields with (18):

$$
\frac{e \hbar}{m_{e} k_{\mathrm{B}} T_{\mathrm{eq}}} \boldsymbol{E}_{\mathrm{ext}} \cdot \boldsymbol{p} f_{1}^{0}\left(E_{p}\right)\left(1-f_{1}^{0}\left(E_{p}\right)\right)=\int \frac{\mathrm{d}^{3} \boldsymbol{p}^{\prime} \Omega_{0}}{(2 \pi)^{3}} w_{\boldsymbol{p} p^{\prime}} f_{1}^{0}\left(E_{p^{\prime}}\right)\left(1-f_{1}^{0}\left(E_{p}\right)\right)\left(\Phi\left(\boldsymbol{p}^{\prime}\right)-\Phi(\boldsymbol{p})\right),
$$

where we have used the assumption that $\Phi(p) \propto E_{\text {ext }}$ and neglected terms with a higher order of $E_{\text {ext }}$ (linearized Boltzmann equation). With the definition of the relaxation time tensor: $\hat{\tau}(\boldsymbol{p})$

$$
\Phi(\boldsymbol{p})=\frac{e \hbar}{m_{e} k_{\mathrm{B}} T_{\mathrm{eq}}} \boldsymbol{E}_{\mathrm{ext}} \cdot \hat{\tau}(\boldsymbol{p}) \cdot \boldsymbol{p}
$$

the equation reads with $\boldsymbol{e}_{E}=\boldsymbol{E}_{\mathrm{ext}} /\left|\boldsymbol{E}_{\mathrm{ext}}\right|$ :

$$
\boldsymbol{e}_{E} \cdot \boldsymbol{p}=\int \frac{\mathrm{d}^{3} \boldsymbol{p}^{\prime} \Omega_{0}}{(2 \pi)^{3}} w_{\boldsymbol{p} \boldsymbol{p}^{\prime}} \frac{f_{1}^{0}\left(E_{p^{\prime}}\right)}{f_{1}^{0}\left(E_{p}\right)} \boldsymbol{e}_{E} \cdot\left[\hat{\tau}\left(\boldsymbol{p}^{\prime}\right) \cdot \boldsymbol{p}^{\prime}-\hat{\tau}(\boldsymbol{p}) \cdot \boldsymbol{p}\right],
$$

which is an equation for $\hat{\tau}(\boldsymbol{p})$, where $\boldsymbol{e}_{E}$ is the unity vector in the direction of the external electric field $\boldsymbol{e}_{E}=E_{\text {ext }} / E_{\text {ext }}$. The electric current density Equation (15) depends only on the deviation of the distribution function since $f_{1}^{0}\left(E_{p}\right)$ is an even function in $p$ (isotropy). We obtain by insertion of Equation (20) into Equation (15):

$$
j=\frac{-e \hbar}{m_{e}} 2 \int \frac{\mathrm{d}^{3} p}{(2 \pi)^{3}} p \Phi(p) f_{1}^{0}\left(E_{p}\right)\left(1-f_{1}^{0}\left(E_{p}\right)\right)
$$


For isotropic systems, we have $\hat{\tau}(\boldsymbol{p})=\tau^{\operatorname{transp}}\left(E_{p}\right)$, so that the solution of Equation (22) is:

$$
\tau^{\operatorname{transp}}\left(E_{p}\right)=\left\{\int \frac{\mathrm{d}^{3} p^{\prime} \Omega_{0}}{(2 \pi)^{3}} w_{p p^{\prime}}(1-\cos \vartheta)\right\}^{-1}
$$

as can be verified by insertion, $\vartheta$ is the angle between $\boldsymbol{p}$ and $\boldsymbol{p}^{\prime}$. The transport relaxation time follows from $\hbar / \tau^{\operatorname{transp}}\left(E_{p}\right)=\pi n_{i} /\left(2 p^{2}\right) \sum_{q} q^{2} V_{\mathrm{ei}}^{2}(q) \delta\left(E_{p+q}-E_{p}\right)$; see [6] and the result below (35).

Now, the conductivity reads with Equation (19):

$$
\sigma=\frac{e^{2} \hbar^{2}}{m_{e}^{2} k_{\mathrm{B}} T_{\mathrm{eq}}} 2 \int \frac{\mathrm{d}^{3} p}{(2 \pi)^{3}} p_{z}^{2} \tau^{\mathrm{transp}}\left(E_{p}\right) f_{1}^{0}\left(E_{p}\right)\left[1-f_{1}^{0}\left(E_{p}\right)\right] .
$$

With (24), we have derived an analytic expression for the conductivity of the Lorentz model solving the Boltzmann equation.

Screened Coulomb interaction. To give explicit expressions, we specify the electron-ion interaction by the screened Coulomb (Debye) interaction:

$$
V_{\mathrm{ei}}^{\mathrm{D}}(\boldsymbol{r})=\frac{e^{2}}{4 \pi \epsilon_{0}|\boldsymbol{r}|} \mathrm{e}^{-\kappa|\boldsymbol{r}|}, \quad \text { with } \kappa^{2}=\frac{e^{2} n_{e}}{\epsilon_{0} k_{\mathrm{B}} T_{\mathrm{eq}}} .
$$

The interaction Hamiltonian $\mathrm{H}^{\prime}$ in the momentum representation is obtained from Fourier transformation with $q=p^{\prime}-p$. It has the matrix elements:

$$
H_{\boldsymbol{p}^{\prime} \boldsymbol{p}}^{\prime}=\frac{1}{\Omega_{0}} \int \mathrm{d}^{3} \boldsymbol{r} \mathrm{e}^{\mathrm{i} \boldsymbol{q} \cdot \boldsymbol{r}} \sum_{i}^{N_{i}} V_{\mathrm{ei}}^{\mathrm{D}}\left(\boldsymbol{r}-\boldsymbol{R}_{i}\right)=-\frac{1}{\Omega_{0}} \sum_{i}^{N_{i}} \mathrm{e}^{\mathrm{i} \boldsymbol{q} \cdot \boldsymbol{R}_{i}} \frac{e^{2}}{\epsilon_{0}\left(q^{2}+\kappa^{2}\right)}
$$

so that $\left|H_{p^{\prime} \boldsymbol{p}}^{\prime}\right|^{2}=S_{\text {ion }}(\boldsymbol{q}) n_{i} / \Omega_{0}\left[e^{2} / \epsilon_{0}\left(q^{2}+\kappa^{2}\right)\right]^{2}$, with the ion structure factor $S_{\text {ion }}(\boldsymbol{q})=$ $\frac{1}{N_{i}} \sum_{i}^{N_{i}} \sum_{j}^{N_{i}} \mathrm{e}^{\mathrm{i} \boldsymbol{q} \cdot\left(\boldsymbol{R}_{i}-\boldsymbol{R}_{j}\right)}$. The inverse relaxation time (24) follows as:

$$
\tau^{-1}\left(E_{p}\right)=n_{i} \frac{1}{4 \pi} \frac{e^{4}}{\epsilon_{0}^{2}} \frac{m_{e}}{\hbar^{3} p^{3}} \Lambda(p)
$$

with the Coulomb logarithm $\Lambda(p)=\int_{0}^{2 p} \frac{1}{\left(q^{2}+\kappa^{2}\right)^{2}} q^{3} \mathrm{~d} q=\ln \sqrt{1+b}-\frac{1}{2} \frac{b}{1+b}, b=4 p^{2} k_{\mathrm{B}} T_{\mathrm{eq}} \epsilon_{0} /\left(e^{2} n_{e}\right)$, where an uncorrelated ion distribution $S_{\text {ion }}(\boldsymbol{q})=1$ is assumed. In the low-density limit at fixed temperature considered here, the Fermi distribution function is replaced by the Boltzmann distribution function. For the conductivity, we finally obtain:

$$
\sigma=\frac{2^{5 / 2}}{\pi^{3 / 2}} \frac{\left(k_{\mathrm{B}} T_{\mathrm{eq}}\right)^{3 / 2}\left(4 \pi \epsilon_{0}\right)^{2}}{m_{e}^{1 / 2} e^{2}} \frac{1}{\Lambda\left(p_{\text {therm }}\right)}=\frac{n_{e} e^{2}}{m_{e}} \bar{\tau}^{\text {transp }}
$$

where the Coulomb logarithm is approximated by the value of the average $p$, with $\hbar^{2} p_{\text {therm }}^{2} / 2 m_{e}=$ $3 k_{\mathrm{B}} T_{\text {eq }} / 2$; see also [6].

Virial expansion. In the more general case where electron-electron collisions are included, we find for the hydrogen plasma $(Z=1)$ the following low-density (virial) expansion [11,12]:

$$
\sigma^{-1}(T, n)=A(T) \ln n+B(T)+C(T) n^{1 / 2} \ln n \pm \ldots
$$

with:

$$
A(T)=-\frac{1}{s} \frac{e^{2} m_{e}^{1 / 2}}{\left(4 \pi \epsilon_{0}\right)^{2}\left(k_{\mathrm{B}} T\right)^{3 / 2}}
$$


For the Lorentz plasma, the value $s=2^{5 / 2} / \pi^{3 / 2}$ is an exact result. It is changed if electron-electron collisions are included, $s=0.591$; see $[4,11-15]$. At low temperatures, the plasma is degenerate, and the Fermi function for the distribution of the electrons cannot be approximated by the Boltzmann distribution function. The value of $s$ is changed and becomes $s=3 /(4 \sqrt{2 \pi})$ for the limit of strong degeneracy. The values of the higher virial coefficients $B(T), C(T)$ are determined by many-body effects and the short-range behavior of the effective interaction. Expressions are given, e.g., in [11], but their exact values are under discussion.

Boltzmann entropy. The Stosszahlansatz of the Boltzmann equation is a semi-empirical assumption, which was highly and controversially discussed for a long time. It makes the Boltzmann equation an irreversible equation of evolution, which is able to describe non-equilibrium processes. It can be shown, see, e.g., [4], that a particular quantity, the Boltzmann entropy:

$$
S^{\text {Boltzmann }}(t)=-k_{\mathrm{B}} \sum_{p} f_{1}(\boldsymbol{p}, t) \ln f_{1}(\boldsymbol{p}, t) \approx-2 k_{\mathrm{B}} \int \frac{\mathrm{d}^{3} p \Omega_{0}}{(2 \pi)^{3}} f_{1}(\boldsymbol{p}, t) \ln f_{1}(\boldsymbol{p}, t)
$$

(classical case), can increase with time and remains constant in thermodynamic equilibrium. It is a main puzzle of nonequilibrium statistical physics how this property can arise on the basis of the reversible equations of motion that define the microscopic approach in physics. It was the merits of Bogoliubov, Zubarev, and others, to give a bridge between both positions, which is presented in Section 4.

It is obvious that the Boltzmann entropy (31) is not the thermodynamic entropy defined by the second law. The evaluation in thermodynamic equilibrium yields in the classical case, where $f_{1}^{0}\left(E_{p}\right) \approx\left(n_{e} / 2\right)\left(2 \pi \hbar^{2} / m_{e} k_{\mathrm{B}} T\right)^{3 / 2} \exp \left(-\hbar^{2} p^{2} / 2 m_{e} k_{\mathrm{B}} T\right)$ (see Equation (14)), the well-known relation:

$$
S^{\text {Boltzmann }}=S_{\text {eq, class }}\left(T, \Omega_{0}, N_{e}\right)=\frac{3}{2} k_{\mathrm{B}} n_{e} \Omega_{0}-n_{e} \Omega_{0} \frac{\mu_{e}}{T}=\frac{U_{\text {class }}}{T}-\mu_{e} \frac{N_{e}}{T}
$$

with $U_{\text {class }}^{\text {id }}=(3 / 2) N_{e} k_{\mathrm{B}} T$ valid for the ideal, noninteracting gas, neglecting the contribution of two-particle correlations, etc. The relation between the Boltzmann entropy and the correct thermodynamic entropy, which takes the correlations in the system into account, becomes clear after introducing the relevant entropy in the subsequent section.

Let us come back to the increase of the Boltzmann entropy. Owing to energy conservation, we have in the external field $\boldsymbol{E}_{\mathrm{ext}}=E_{\mathrm{ext}} \boldsymbol{e}_{z}$ the increase of internal energy of the electron system $\mathrm{d} U_{\text {class }} / \mathrm{d} z=$ $e N_{e} E_{\text {ext }}$ if we shift all electrons by $\mathrm{d} z$. The Boltzmann entropy changes as $\mathrm{d} S^{\text {Boltzmann }} / \mathrm{d} t=e N_{e} E_{\text {ext }} v / T$ where $v$ is the mean velocity of the electron system. This value coincides with the imported power $\mathrm{d} U_{\text {class }} / \mathrm{d} t=\Omega_{0} j \cdot E_{\text {ext }}$. The chemical potential $\mu_{e}$, which is connected with the electron density, remains constant. If we assume that by reason of any unknown strong relaxation process, the electron system remains near the thermodynamic equilibrium, and the change of internal energy $U_{\text {class }}$ is described by the temperature $T$. Then, the change of the temperature would be:

$$
\frac{\mathrm{d} T}{\mathrm{~d} t}=\frac{2}{3 k_{\mathrm{B}} n_{e}} j \cdot E_{\mathrm{ext}}=\frac{2}{3 k_{\mathrm{B}} n_{e}} \sigma E_{\mathrm{ext}}^{2} .
$$

However, the properties of the open system are not time dependent in the stationary case. Because the energy balance is of second order in $E_{\text {ext }}$, it is neglected in linear response theory. Nevertheless, it is not clear whether the kinetic energy of moving bodies can be denoted as heat and interpreted as entropy, for instance considering the motion of celestial bodies.

Energy export to ions. To have a stationary state, the gain of internal energy must be transferred to the bath. We mentioned already the emission and absorption of photons; see Equation (11) and Section 8 below. Another microscopic model for the energy transfer is the collision with ions. Kinetic theory describes not only the dissipation of the total electron momentum, but also the transfer of the kinetic energy of electrons to the ion subsystem. We consider ions with finite mass $M_{i}$ so that 
recoil effects are possible at collisions, which are no longer elastic. A second model is the excitation of collective modes of the ion system, the phonons, which may give a different picture.

Considering the energy transfer to the ions, we have a two-temperature situation with $T_{e}>T_{i}$. According to Landau and Spitzer [15], the energy density $e_{\text {kin }}=(3 / 2) n_{e} k_{\mathrm{B}} T_{e}$ is decreased as:

$$
\frac{\mathrm{d}}{\mathrm{d} t} e_{\text {kin }}=\frac{3}{2} n_{e} k_{\mathrm{B}}\left(T_{e}-T_{i}\right) \frac{m_{e}}{M_{i} \bar{\tau}}
$$

with the relaxation time (slightly different from the transport relaxation time (24)),

$$
\bar{\tau}=\frac{\beta \hbar^{2}}{6 n_{e} \Omega_{0}} \sum_{p} p^{2} \tau\left(E_{p}\right) f_{1}^{0}\left(E_{p}\right)\left[1-f_{1}^{0}\left(E_{p}\right)\right],
$$

with $\hbar / \tau\left(E_{p}\right)=2 \pi n_{i} \sum_{q} V_{\mathrm{ei}}^{2}(q) \delta\left(E_{p+q}-E_{p}\right)$; see [6]. This compensates the gain $n_{e} e^{2} E_{\mathrm{ext}}^{2} \bar{\tau} / m_{e}$ so that:

$$
k_{\mathrm{B}}\left(T_{e}-T_{i}\right)=\frac{2 M_{i}}{3 m_{e}^{2}} \bar{\tau}^{2} e^{2} E_{\mathrm{ext}}^{2} .
$$

As already mentioned, the difference is of second order in $E_{\text {ext }}$ and may be neglected in linear response theory, but it becomes large in the adiabatic limit $m_{e} / M_{i} \rightarrow 0$. The exact value of the energy current may be changed within a more detailed description, but these arguments remain. Furthermore, the correlations in the ion system given by the pair distribution function, as well as collective modes in the excitation spectrum leading to dressed states will change the magnitude of the energy transfer to the (ionic) bath.

\section{The Zubarev Method of Nonequilibrium Statistical Operator}

The von Neumann equation. Whereas the Boltzmann equation used the semiempirical "Stosszahlansatz", the systematic derivation of the kinetic equation for $f_{1}(\boldsymbol{p}, t)$ from a microscopic description was intended by Bogoliubov [10] using the principle of weakening of initial correlations. A more general formulation of this important step to work out the theory of non-equilibrium processes was given by Zubarev [1-3]. To calculate averages $\langle A\rangle^{t}=\operatorname{Tr}\{\rho(t) A\}$, we need the statistical operator $\rho(t)=\sum_{n}\left|\psi_{n}(t)\right\rangle w_{n}\left\langle\psi_{n}(t)\right|$, which describes the probability distribution $w_{n}$ of microstates $\left|\psi_{n}(t)\right\rangle$ in the thermodynamic macrostate. Let us assume that the equation of motion of each realization, quantum state $\left|\psi_{n}(t)\right\rangle$, is given by the reversible Schrödinger equation i $\hbar \partial|\psi(t)\rangle / \partial t=\mathrm{H}^{t}|\psi(t)\rangle$. The Hamiltonian $\mathrm{H}^{t}$ may contain time-dependent external fields (for an isolated system in equilibrium, usually, the energy eigenfunctions are identified with the eigenstates of $\rho$, and the time evolution refers only to the phase of $\left.\left|\psi_{n}(t)\right\rangle\right)$. Then, the von Neumann equation follows as the equation of motion for the statistical operator,

$$
\frac{\partial}{\partial t} \rho(t)+\frac{\mathrm{i}}{\hbar}\left[\mathrm{H}^{t}, \rho(t)\right]=0 .
$$

Despite its character as a fundamental equation of motion in statistical physics, the von Neumann equation has two shortcomings:

(i) To determine $\rho(t)$, the initial value problem has to be solved.

(ii) As a reversible equation of motion, it cannot describe irreversible processes.

The solution of (i) is known for thermodynamic equilibrium, where $\rho(t)=\rho_{\text {eq }}$ does not depend on time. We need an additional principle to determine $\rho_{\mathrm{eq}}$, the maximum of information entropy:

$$
S_{\text {inf }}[\rho]=-\operatorname{Tr}\{\rho \ln \rho\}
$$


for arbitrary $\rho$ that are consistent with the given conditions $\operatorname{Tr}\{\rho\}=1$ (normalization) and the given constants of motion $\mathrm{C}_{n}$ :

$$
\operatorname{Tr}\left\{\rho \mathrm{C}_{n}\right\}=\left\langle\mathrm{C}_{n}\right\rangle .
$$

These are external conditions that describe how we influence the system, for instance preparing the volume and particle number and coupling this to a thermal bath. These self-consistency conditions can be implemented in the variational principle using the method of Lagrange multipliers; see [1]. The corresponding maximum value for $S_{\text {inf }}[\rho]$ :

$$
S_{\mathrm{eq}}\left[\rho_{\mathrm{eq}}\right]=-k_{\mathrm{B}} \operatorname{Tr}\left\{\rho_{\mathrm{eq}} \ln \rho_{\mathrm{eq}}\right\}
$$

is the equilibrium entropy of the system at given constraints $\left\langle\mathrm{C}_{n}\right\rangle$, and $k_{\mathrm{B}}$ is the Boltzmann constant.

Well-known solutions of this variational principle are the Gibbs ensembles for thermodynamic equilibrium. For instance, in the grand canonical ensemble, the average value of energy is realized by a Lagrange multiplier, which is identified as temperature $T$, and the average value of particle numbers $N_{c}$ is realized by Lagrange multipliers, which are identified as chemical potentials $\mu_{c}$. The Lagrange multipliers are eliminated by solving Equation (39). The corresponding relations are known as the equations of state.

With respect to Item (ii), the expression $\operatorname{Tr}\{\rho(t) \ln \rho(t)\}$ cannot be used to define the entropy in non-equilibrium, because it cannot increase with time. Using the von Neumann equation,

$$
\frac{\mathrm{d}}{\mathrm{d} t}[\operatorname{Tr}\{\rho(t) \ln \rho(t)\}]=0
$$

follows. The discrepancy with the second law of thermodynamics that entropy may increase with time for a system in nonequilibrium can be solved according to Zubarev by a modification of the von Neumann equation.

The relevant statistical operator. Zubarev [1] proposed to extend the concept of information theory also to construct a relevant statistical operator $\rho_{\text {rel }}(t)$ for given averages of relevant observables $\left\{B_{n}\right\}$ that are not constants of motion, but may change with time. Now, at each time step $t$, we find the maximum of information entropy solving:

$$
-\delta\left[\operatorname{Tr}\left\{\rho_{\text {rel }}(t) \ln \rho_{\text {rel }}(t)\right\}\right]=0
$$

with the self-consistency conditions:

$$
\operatorname{Tr}\left\{\rho_{\text {rel }}(t) \mathrm{B}_{n}\right\} \equiv\left\langle\mathrm{B}_{n}\right\rangle_{\text {rel }}^{t}=\left\langle\mathrm{B}_{n}\right\rangle^{t} .
$$

and $\operatorname{Tr}\left\{\rho_{\text {rel }}(t)\right\}=1$. We use time-dependent Lagrange multipliers $\lambda_{n}(t)$ to account for the self-consistency conditions (43). The solution of the variational problem is the generalized Gibbs distribution:

$$
\rho_{\text {rel }}(t)=\mathrm{e}^{-\Phi(t)-\sum_{n} \lambda_{n}(t) \mathrm{B}_{n}}, \quad \Phi(t)=\ln \operatorname{Tr}\left\{\mathrm{e}^{-\sum_{n} \lambda_{n}(t) \mathrm{B}_{n}}\right\},
$$

where, as in the equilibrium case, the Lagrange multipliers $\lambda_{n}(t)$ (thermodynamic parameters) are determined by the self-consistency conditions (43) and have to be eliminated. With the thermodynamic potential $\Phi(t)$ (Massieux-Planck function), the normalization condition is realized.

In the generalization of the equilibrium cases, the maximum of information entropy can be considered as the relevant entropy in nonequilibrium:

$$
S_{\text {rel }}(t)=-k_{\mathrm{B}} \operatorname{Tr}\left\{\rho_{\text {rel }}(t) \ln \rho_{\text {rel }}(t)\right\} .
$$


Maxwell relations similar to the relations known from equilibrium thermodynamics can be derived [1]. In addition, we find for the production of relevant entropy (see also [4]),

$$
\frac{\mathrm{d} S_{\text {rel }}(t)}{\mathrm{d} t}=\sum_{n} \lambda_{n}(t) \frac{\mathrm{i}}{\hbar}\left\langle\left[\mathrm{H}^{t}, \mathrm{~B}_{n}\right]\right\rangle^{t}=\sum_{n} \lambda_{n}(t)\left\langle\dot{\mathrm{B}}_{n}\right\rangle^{t} .
$$

This relation is well known from the thermodynamics of irreversible processes. In contrast to Equation (41), this expression can have a positive value so that $S_{\text {rel }}(t)$ can increase with time.

Coming back to the electrical conductivity as an example for an irreversible process, the nonequilibrium state is characterized by the current density $j$, and with the density of heat production $j \cdot E_{\mathrm{ext}}=\sigma \boldsymbol{E}_{\mathrm{ext}}^{2}$, Equation (2), the density of entropy production is given by:

$$
\frac{\mathrm{d} S_{\text {rel }}(t)}{\mathrm{d} t}=\frac{\Omega_{0}}{T} \boldsymbol{j} \cdot \boldsymbol{E}_{\mathrm{ext}}=\frac{\Omega_{0}}{T} \sigma \boldsymbol{E}_{\mathrm{ext}}^{2} .
$$

This result is obtained if the position $\mathbf{R}$ of the electrons, which couples to the external field, is considered as a relevant observable, so that $\langle\dot{\mathbf{R}}\rangle=\left(\hbar / m_{e}\right)\langle\mathbf{P}\rangle=-\left(\Omega_{0} / e\right) j$. The Hamiltonian contains also the external field $\boldsymbol{E}_{\text {ext }}$, which must be compensated to obtain the stationary, homogeneous case. For this, the value $\vec{\lambda}=\beta E_{\text {ext }}$ is needed. It acts like a position-dependent chemical potential $\mu_{\text {rel }}(\boldsymbol{r})$, which couples to the local electron density. Other choices of relevant observables are given below in Section 5 .

A well-known example is the Boltzmann entropy (31). This expression is the relevant entropy in nonequilibrium if the single-particle distribution function $f_{1}(p, t)$ is considered as a relevant observable. It can increase with time, as proven by the famous H-theorem. However, it is not the correct thermodynamic entropy, because in thermodynamic equilibrium, the contribution of correlations to the potential energy is missing.

The introduction of the relevant statistical operator does not solve the problem of nonequilibrium statistical physics. It is a semiempirical approach, and the selection of the set of relevant observables $\left\{\mathrm{B}_{n}\right\}$ is arbitrary, but determines the result for the relevant entropy. $S_{\text {rel }}(t)$ is not the thermodynamic entropy because it is based on the arbitrary choice of the set $\left\{B_{n}\right\}$ of relevant observables, and not all possible variables are correctly reproduced. The possible increase of the relevant entropy with time (47) is the effect of coarse graining introducing the reduced set of relevant observables. A main deficit is that it does not respect the equations of motion; it does not obey the Liouville-von Neumann equation. The dependence on time is parametric, but not dynamic. An important step to solve the problem of dynamics is given by the Zubarev method of the Nonequilibrium Statistical Operator (NSO).

The Zubarev solution of the initial value problem. The formal solution of the von Neumann Equation (37) is easily found,

$$
\rho(t)=\mathrm{U}\left(t, t_{0}\right) \rho\left(t_{0}\right) \mathrm{U}^{\dagger}\left(t, t_{0}\right) .
$$

The unitary time evolution operator $\mathrm{U}\left(t, t_{0}\right)$ is the solution of the differential equation:

$$
\mathrm{i} \hbar \frac{\partial}{\partial t} \mathrm{U}\left(t, t_{0}\right)=\mathrm{H}^{t} \mathrm{U}\left(t, t_{0}\right),
$$

with the initial condition $\mathrm{U}\left(t_{0}, t_{0}\right)=1$. If the Hamiltonian is not time dependent, we have:

$$
\mathrm{U}\left(t, t_{0}\right)=\mathrm{e}^{-\frac{\mathrm{i}}{\hbar} \mathrm{H}\left(t-t_{0}\right)} .
$$

If the Hamiltonian $\mathrm{H}^{t}$ is time dependent, the solution is given by a time-ordered exponent.

However, we do not know the initial state $\rho\left(t_{0}\right)$. An answer was given by Zubarev [1]. In the first step, we can take instead $\rho\left(t_{0}\right)$ the relevant statistical operator $\rho_{\text {rel }}\left(t_{0}\right)$ at some initial time $t_{0}$,

$$
\rho_{t_{0}}(t)=\mathrm{U}\left(t, t_{0}\right) \rho_{\text {rel }}\left(t_{0}\right) \mathrm{U}^{\dagger}\left(t, t_{0}\right) .
$$


According to the Bogoliubov principle of the weakening of initial correlations [10], the missing correlations that are not correctly implemented in $\rho_{\text {rel }}\left(t_{0}\right)$ are produced by the dynamical evolution of the system. This procedure is well known from molecular dynamics simulations where, starting from an artificial initial configuration, the stationary distribution is approximated after an initial relaxation time (synchronization). The crucial point is that one should consider the limit $t_{0} \rightarrow-\infty$ so that enough time is available to establish all missing correlations. As known from ergodic theory, at least the conserved observables have to be correctly implemented in $\rho_{\text {rel }}\left(t_{0}\right)$ because they cannot be produced dynamically. The more observables $\left\{\mathrm{B}_{n}\right\}$ are correctly described by $\rho_{\text {rel }}\left(t_{0}\right)$, the less time is necessary to produce the remaining correlations. Notice that the self-consistency conditions (43) valid at $t_{0}$ are not automatically valid also at $t$ averaging with $\rho_{t_{0}}(t)$, if the time evolution according to Equation (51) is taken. Below, we discuss the case of incomplete dynamics if the open system is in contact with a bath.

In the second step, instead of selecting a special instant of time $t_{0}$, the average over the past is performed. According to Abel's theorem (see [1-3]), the limit $t_{0} \rightarrow-\infty$ can be replaced by the limit $\epsilon \rightarrow+0$ in the expression:

$$
\rho_{\epsilon}(t)=\epsilon \int_{-\infty}^{t} \mathrm{e}^{\epsilon\left(t_{1}-t\right)} \mathrm{U}\left(t, t_{1}\right) \rho_{\text {rel }}\left(t_{1}\right) \mathrm{U}^{\dagger}\left(t, t_{1}\right) \mathrm{d} t_{1} .
$$

This averaging over different initial time instants means a mixing of phases so that long-living oscillations are damped out. Finally, we obtain the nonequilibrium statistical operator as:

$$
\rho_{\mathrm{NSO}}(t)=\lim _{\epsilon \rightarrow 0} \rho_{\epsilon}(t) .
$$

This way, $\rho_{\text {rel }}\left(t_{1}\right)$ for all times $-\infty<t_{1}<t$ serves as the initial condition to solve the Liouville-von Neumann equation according to the Bogoliubov principle of weakening of initial correlations. The past that is of relevance, given by the relaxation time $\tau$, becomes shorter, if the relevant (long-living) correlations are already correctly implemented. The limit $\epsilon \rightarrow+0$ is to be considered as $\epsilon \ll 1 / \tau$. The limit $\epsilon \rightarrow+0$ has to be performed after the thermodynamic limit.

Selection of the set of relevant observables. An open issue is the appropriate selection of the set of relevant observables $\left\{B_{n}\right\}$ to characterize the nonequilibrium state of the system. The method of the nonequilibrium statistical operator allows one to extend the set of relevant observables arbitrarily so that the choice of the set of relevant observables seems to be irrelevant. All missing correlations are produced dynamically. As a minimum, the constants of motion $C_{n}$ have to be included because their relaxation time is infinite, and their averages cannot be produced dynamically. The resulting $\rho_{\mathrm{NSO}}(t)$ (53) should not depend on the (arbitrary) choice of relevant observables $\left\{\mathrm{B}_{n}\right\}$ if the limit $\epsilon \rightarrow 0$ is correctly performed. However, usually perturbation theory is applied, so that the result will depend on the selection of the set of relevant observables. The inclusion of long-living correlations into $\left\{B_{n}\right\}$ allows one to use lower order perturbation expansions to obtain acceptable results. In the context with the electrical conductivity, the selection of different sets of relevant observables has been extensively discussed; see, e.g., [4-6,11-14,16].

Entropy in the Zubarev NSO approach. An intricate problem is the definition of entropy for the nonequilibrium state. In nonequilibrium, entropy is produced, as investigated in the phenomenological approach to the thermodynamics of irreversible processes, considering currents induced by the generalized forces. Such a behavior occurs for the relevant entropy defined by the relevant distribution (45). A famous example that shows the increase of the relevant entropy (31) with time is the Boltzmann $\mathrm{H}$ (capital eta) theorem, where the relevant observables to define the nonequilibrium state is the single particle distribution function.

Note that the entropy puzzle cannot be solved by the relevant entropy. Not only the well-defined thermodynamic entropy in equilibrium is not reproduced. A so-called coarse graining has been performed. The information about the state is reduced because the degrees of freedom to describe the system are reduced. This may be an averaging in phase space over small cells. Furthermore, 
the average over different phases of the quantum state, the destruction of quantum interference (dephasing), and other projection techniques will destroy information. The loss of information then gives the increase of entropy. This procedure is artificial, depending on our way of describing the details of a process, or anthropomorphic, related to our technical possibilities to prepare and measure the state of a system and control the dynamics. In certain situations, such as quantum master equations (see [17,18]), kinetic theory (see [16]), and linear response theory (see [19]), the choice of relevant observables becomes quite natural (see also [4]). In general, there is no first principle approach that gives the decision about how the relevant degrees of freedom have to be selected out. From a fundamental point of view, this situation is unsatisfactory.

A possible definition of the entropy would be:

$$
S_{\mathrm{NSO}}(t)=-k_{\mathrm{B}} \operatorname{Tr}\left\{\rho_{\mathrm{NSO}}(t) \ln \rho_{\mathrm{NSO}}(t)\right\} .
$$

It is an open question whether the entropy $S_{\mathrm{NSO}}(t)$ will increase also in the limit $\epsilon \rightarrow+0$. Coming back to our example of DC conductivity, the stationary state means that $\rho_{\mathrm{NSO}}(t)$ should not depend on time $t$. The entropy in the open system is constant, but there is also a constant production of entropy, which is not derived from (54).

The extended Liouville-von Neumann equation. We consider a closed system with known dynamics $\mathrm{H}^{t}$. The nonequilibrium statistical operator $\rho_{\epsilon}(t)$, Equation (52), obeys the extended von Neumann equation:

$$
\frac{\partial \rho_{\epsilon}(t)}{\partial t}+\frac{\mathrm{i}}{\hbar}\left[\mathrm{H}^{t}, \rho_{\epsilon}(t)\right]=-\epsilon\left(\rho_{\epsilon}(t)-\rho_{\text {rel }}(t)\right) .
$$

as can be seen after simple derivation with respect to time. In contrast to the von Neumann Equation (37), a source term arises on the right-hand side that becomes infinitesimally small in the limit $\epsilon \rightarrow+0$. This source term breaks the time inversion symmetry so that, for any finite value of $\epsilon$, the solution $\rho_{\epsilon}(t)$ describes in general an irreversible evolution with time.

The source term implements the "initial condition" in the equation of motion as expressed by $\rho_{\text {rel }}(t)$. Formally, the source term looks like a relaxation process. In addition to the internal dynamics, the system evolves towards the relevant distribution.

The construction of the source term is such that the time evolution of the relevant variables is not affected by the source term (we use the invariance of the trace with respect to cyclic permutations),

$$
\frac{\mathrm{d}}{\mathrm{d} t}\left\langle\mathrm{~B}_{n}\right\rangle^{t}=\operatorname{Tr}\left\{\frac{\partial \rho_{\epsilon}(t)}{\partial t} \mathrm{~B}_{n}\right\}=-\operatorname{Tr}\left\{\frac{\mathrm{i}}{\hbar}\left[\mathrm{H}^{t}, \rho_{\epsilon}(t)\right] \mathrm{B}_{n}\right\}-\epsilon\left[\left\langle\mathrm{B}_{n}\right\rangle^{t}-\left\langle\mathrm{B}_{n}\right\rangle_{\text {rel }}^{t}\right]=\left\langle\frac{\mathrm{i}}{\hbar}\left[\mathrm{H}^{t}, \mathrm{~B}_{n}\right]\right\rangle^{t}=\left\langle\dot{\mathrm{B}}_{n}\right\rangle^{t} .
$$

The source term cancels because of the self-consistency conditions (43). Thus, the time evolution of the relevant observables satisfies the dynamical equations of motion according to the Hamiltonian $\mathrm{H}^{t}$.

Any real system is in contact with the surroundings. The intrinsic dynamics described by the Hamiltonian $\mathrm{H}^{t}$ is modified owing to the coupling of the open system to the bath. Within the quantum master equation approach (see Section 6 below), we can approximate the influence term describing the coupling to the bath by a relaxation term similar to the source term. However, at present, we consider the source term as a purely mathematical tool to select the retarded solution of the Liouville-von Neumann equation, and physical results are obtained only after performing the limit $\epsilon \rightarrow 0$.

\section{Generalized Linear Response Theory}

Linearization of the NSO. We use the Zubarev NSO method to calculate the electrical conductivity. It unifies kinetic theory and linear response theory. An extended discussion of this generalized linear response theory can be found in the literature $[4,6,11,13,14,16]$, which will not be repeated here.

The main idea is to consider small fluctuations near the thermodynamic equilibrium. In the relevant statistical operator (44) containing the observables $B_{n}$ with the Lagrange parameters $\lambda_{n}(t)$, we extract the conserved observables $C_{n}$ with the Lagrange parameters $\beta, \mu_{e}$, which determine 
the thermodynamic equilibrium. The remaining observables with Lagrange parameters $\beta F_{n}(t)$ are considered as small fluctuations, so that we can expand with respect to $F_{n}(t)$. In first order, we have:

$$
\rho_{\text {rel }}(t)=\rho_{\text {eq }}+\beta \int_{0}^{1} \mathrm{~d} \lambda \sum_{n} F_{n}(t) \mathrm{B}_{n}(\mathrm{i} \hbar \beta \lambda) \rho_{\mathrm{eq}} .
$$

Here, we made use of the modified Heisenberg picture $\mathrm{O}(\tau)=\exp (\mathrm{i} \mathcal{H} \tau / \hbar) \mathrm{O} \exp (-\mathrm{i} \mathcal{H} \tau / \hbar)$ with $\tau \rightarrow \mathrm{i} \hbar \beta \lambda$ and replacing in the exponents $\mathrm{H}_{\mathrm{S}}$ by $\mathcal{H}=\mathrm{H}_{\mathrm{S}}-\mu_{e} \mathrm{~N}_{e}$. Note that $\mathcal{H}$ does not contain the external field $\mathrm{H}_{\mathrm{F}}^{t}$. Because of homogeneity, in $\rho_{\text {rel }}$, any position-dependent external field has to be compensated by a position-dependent chemical potential.

After integration by parts, the NSO (52) with $\mathrm{H}^{t}=\mathrm{H}_{\mathrm{S}}+\mathrm{H}_{\mathrm{F}}^{t}$ has the form:

$$
\rho_{\epsilon}(t)=\rho_{\text {rel }}(t)-\int_{-\infty}^{t} \mathrm{~d} t_{1} \mathrm{e}^{\epsilon\left(t_{1}-t\right)} \mathrm{U}\left(t, t_{1}\right)\left\{\frac{\mathrm{i}}{\hbar}\left[\left(\mathrm{H}_{\mathrm{S}}+\mathrm{H}_{\mathrm{F}}^{t_{1}}\right), \rho_{\text {rel }}\left(t_{1}\right)\right]+\frac{\partial}{\partial t_{1}} \rho_{\text {rel }}\left(t_{1}\right)\right\} \mathrm{U}^{\dagger}\left(t, t_{1}\right) .
$$

In our case of DC conductivity, where $\mathrm{H}_{\mathrm{F}}^{t}=e \boldsymbol{E}_{\mathrm{ext}} \cdot \mathbf{R}(5)$, we expand up to first order in $E_{\mathrm{ext}}$ and assume $F_{n} \propto E_{\text {ext }}$. Since $H_{S}$ commutes with the equilibrium $\rho_{\text {eq, }}$, the curly bracket is of order $\mathcal{O}\left(E_{\text {ext }}\right)$. In the stationary state, the statistical operators are not depending on $t$. With $(\mathrm{i} / \hbar)\left[\mathrm{H}_{\mathrm{S}}, \mathbf{R}\right]=\mathbf{P} / m_{e}$, we arrive at (in this section, we use the notation $\mathbf{P}=\sum_{i}^{N_{e}} \hbar \mathbf{p}_{i}$ for the total momentum):

$$
\rho_{\epsilon}=\rho_{\text {rel }}-\beta \int_{-\infty}^{0} \mathrm{~d} t_{1} \mathrm{e}^{\epsilon t_{1}} \int_{0}^{1} \mathrm{~d} \lambda\left[-\frac{e}{m_{e}} \boldsymbol{E}_{\mathrm{ext}} \cdot \mathbf{P}\left(\mathrm{i} \lambda \beta \hbar+t_{1}\right) \rho_{\mathrm{eq}}+\sum_{n} F_{n} \dot{\mathrm{B}}_{n}\left(\mathrm{i} \lambda \beta \hbar+t_{1}\right) \rho_{\mathrm{eq}}\right] .
$$

In the stationary case considered here, there is no dependence of $E_{\text {ext }}(t), \rho_{\epsilon}(t), \rho_{\text {rel }}(t), F_{n}(t)$ on time $t$. After fully linearizing the statistical operator (59) with (57), we have for the electrical current density:

$$
j=\frac{e}{m_{e} \Omega_{0}}\langle\mathbf{P}\rangle=\frac{e \beta}{m_{e} \Omega_{0}}\left\{\sum_{n}\left[\left(\mathbf{P} \mid \mathrm{B}_{n}\right)-\left\langle\mathbf{P} ; \dot{\mathrm{B}}_{n}\right\rangle_{i \epsilon}\right] F_{n}+\langle\mathbf{P} ; \mathbf{P}\rangle_{i \epsilon} \cdot \frac{e}{m_{e}} \boldsymbol{E}_{\text {ext }}\right\}=\sigma \boldsymbol{E}_{\text {ext }} .
$$

Here, we introduced the Kubo scalar product (the particle number commutes with the observables):

$$
(\mathrm{A} \mid \mathrm{B})=\int_{0}^{1} \mathrm{~d} \lambda \operatorname{Tr}\left\{\mathrm{A} \mathrm{e}^{-\lambda \beta \mathcal{H}} \mathrm{B} \mathrm{e}^{\lambda \beta \mathcal{H}} \rho_{\mathrm{eq}}\right\}=\int_{0}^{1} \mathrm{~d} \lambda \operatorname{Tr}\left\{\mathrm{AB}(\mathrm{i} \lambda \beta \hbar) \rho_{\mathrm{eq}}\right\},
$$

and its Laplace transform, the thermodynamic correlation function:

$$
\langle\mathrm{A} ; \mathrm{B}\rangle_{z}=\int_{-\infty}^{0} \mathrm{~d} t \mathrm{e}^{-\mathrm{i} z t}(\mathrm{~A} \mid \mathrm{B}(t))=\int_{0}^{\infty} \mathrm{d} t \mathrm{e}^{\mathrm{i} z t}(\mathrm{~A}(t) \mid \mathrm{B}) .
$$

Note that similar expressions can be given for time-dependent (periodic) fields [4,16]. In the classical limit where the variables commute, additional integrals expanding the exponential are avoided.

Kubo formula. In particular, for the empty set of relevant fluctuations $\left\{B_{n}\right\}$ so that $\rho_{\text {rel }}=\rho_{\text {eq, }}$, we obtain the Kubo formula (we choose $j, \boldsymbol{E}_{\text {ext }}$ parallel to the $z$ axis so that $\mathbf{P}=\mathbf{P} \cdot \boldsymbol{e}_{z}$ is the $z$ component of $\mathbf{P}$ ):

$$
\sigma_{\mathrm{DC}}^{\mathrm{Kubo}}=\frac{e^{2} \beta}{m_{e}^{2} \Omega_{0}}\langle\mathrm{P} ; \mathrm{P}\rangle_{\mathrm{i} \epsilon} .
$$


A similar expression can also be given for the dynamical, wave-number vector-dependent conductivity $\sigma(\boldsymbol{q}, \omega)$, which is related to other quantities such as the response function, the dielectric function, or the polarization function (see $[4,14,16,20])$. The relation (63) is a special form of the Fluctuation-Dissipation Theorem (FDT), which connects the time evolution of equilibrium fluctuations, here the current, with transport coefficients, here the conductivity.

In the lowest order of perturbation theory, we have the result:

$$
\sigma_{\mathrm{DC}}^{\mathrm{Kubo}, 0}=\frac{n_{e} e^{2}}{m_{e}} \frac{1}{\epsilon}
$$

which diverges in the limit $\epsilon \rightarrow 0$. Perturbation theory cannot be applied immediately to evaluate the DC conductivity for interacting charged particles. The use of perturbation theory for the Kubo formula and performing partial summations are discussed in [6]. To avoid perturbation theory, the Kubo formula can be evaluated numerically, e.g., Molecular Dynamics (MD) simulations. Most recent approaches use the Kubo-Greenwood formula [6] and treat the electron system via Density-Functional Theory (DFT). In this approach also, an $\epsilon$-broadening of the $\delta$-like contributions is needed.

Elimination of the Lagrange parameters $F_{n}$. The Lagrange parameters $F_{n}$ must be eliminated with Equation (43). After linearization (59), we find the response equations:

$$
\left\langle\mathrm{B}_{m}\right\rangle-\left\langle\mathrm{B}_{m}\right\rangle_{\text {rel }}=-\sum_{n}\left\langle\mathrm{~B}_{m} ; \dot{\mathrm{B}}_{n}\right\rangle_{i \epsilon} F_{n}+\left\langle\mathrm{B}_{m} ; \mathrm{P}\right\rangle_{i \epsilon} \frac{e}{m_{e}} E_{\text {ext }}=0
$$

to determine the response parameters $F_{n}$, and the number of equations coincides with the number of variables to be determined. The coefficients of this linear system:

$$
\sum_{n} P_{m n} F_{n}=D_{m} E_{\mathrm{ext}}
$$

of equations are given by equilibrium correlation functions. Using Cramer's rule, the response parameters $F_{n}$ are found to be proportional to the external field $E_{\text {ext }}$ with coefficients that are ratios of two determinants. The evaluation of the matrix elements that are equilibrium correlation functions can be performed using different methods such as numerical simulations, quantum statistical perturbation theories such as thermodynamic Green functions and Feynman diagrams, path integral methods, etc. Simple expressions for the conductivity are obtained if $\mathbf{P}$ is included in the set of relevant observables $\left\{\mathrm{B}_{n}\right\}$.

Force-force correlation function. The nonequilibrium state is characterized by the electrical current density $j=e /\left(m_{e} \Omega_{0}\right)\langle\mathrm{P}\rangle$, which is related to the total momentum $\mathrm{P}=\hbar \sum_{i} \mathrm{p}_{i, z}$. This motivates selecting it as the relevant observable $\mathrm{B}_{n} \rightarrow \mathrm{P}$. After the solution of the response equation and performing partial integrations [4,16], the resistance $R$ in the static limit follows as [21]:

$$
R=\frac{1}{\sigma}=\frac{\Omega_{0} \beta}{e^{2} N_{e}^{2}} \frac{\langle\dot{\mathrm{P}} ; \dot{\mathrm{P}}\rangle_{\mathrm{i} \epsilon}}{1+\frac{\beta}{m_{e} N_{e}}\langle\mathrm{P} ; \dot{\mathrm{P}}\rangle_{\mathrm{i} \epsilon}}=\frac{\Omega_{0} \beta}{e^{2} N_{e}^{2}}\left\langle\dot{\mathrm{P}}_{\mathrm{st}} ; \dot{\mathrm{P}}_{\mathrm{st}}\right\rangle_{\mathrm{i} \epsilon}
$$

where $\dot{\mathrm{P}}_{\mathrm{st}}=\dot{\mathrm{P}}-\frac{\langle\dot{\mathrm{P}} ; \mathrm{P}\rangle_{\mathrm{i} \epsilon}}{\langle\mathrm{P} ; \mathrm{P}\rangle_{\mathrm{i} \epsilon}}$ is the stochastic part of the force, which is orthogonal (independent) on $\mathrm{P}$ (cf. the Langevin approach to Brownian motion), $\left\langle\dot{\mathrm{P}}_{\mathrm{st}} ; \mathrm{P}\right\rangle_{\mathrm{i} \epsilon}=0$. According to the so-called second fluctuation-dissipation theorem, the resistivity is given by the equilibrium correlation function of stochastic forces.

Now, perturbation theory can be applied, and in Born approximation, the Ziman formula, a standard result of transport theory, is obtained. We conclude that the use of relevant observables gives a better starting point for perturbation theory. In contrast to the Kubo formula that starts from 
thermal equilibrium as the initial state, the correct current is already reproduced in the initial state and must not be created by the dynamical evolution.

We give the result for the force-force correlation function in Born approximation:

$$
\begin{aligned}
\langle\dot{P} ; \dot{\mathrm{P}}\rangle_{\mathrm{i} \epsilon} & =-\sum_{p, p^{\prime}, \boldsymbol{q}, \boldsymbol{q}^{\prime}} \int_{-\infty}^{0} \mathrm{~d} t \mathrm{e}^{\epsilon t} \int_{0}^{1} \mathrm{~d} \lambda \mathrm{e}^{\frac{\mathrm{i}}{\hbar}\left(E_{p}-E_{p+q}\right)(t-\mathrm{i} \hbar \beta \lambda)} V_{q} V_{q^{\prime}} q_{z} q_{z}^{\prime}\left\langle\mathrm{a}_{p+q}^{+} \mathrm{a}_{p} \mathrm{a}_{\boldsymbol{p}^{\prime}+\boldsymbol{q}^{\prime}}^{+} \mathrm{a}_{\boldsymbol{p}^{\prime}}\right\rangle \mathrm{eq} \\
& =\sum_{p, q}\left|V_{q}\right|^{2} \delta\left(E_{p}-E_{p+q}\right) f_{p}\left(1-f_{p}\right) \pi \hbar \frac{q^{2}}{3} .
\end{aligned}
$$

For the Debye potential, we obtain the result (28), but with the prefactor $s=3 /(4 \sqrt{2 \pi})$.

Despite the excellent results using the Ziman formula in solid and liquid metals where the electrons are strongly degenerate, we cannot conclude that the result (67) with (68) for the conductivity is already correct for low-density plasmas (the non-degenerate limit if $T$ remains constant) in the lowest order of perturbation theory considered here. The prefactor $s=3 /(4 \sqrt{2 \pi})$ is wrong. If we go to the next order of interaction, divergent contributions arise. These divergences can be avoided by performing a partial summation, which will also change the coefficients in Equation (29), which are obtained in the lowest order of the perturbation expansion. The divergent contributions can also be avoided extending the set of relevant observables $\left\{\mathrm{B}_{n}\right\}$ (see Ref. [11]).

Higher moments of the single-particle distribution function. Besides the electrical current, also other deviations from thermal equilibrium can occur in the stationary nonequilibrium state such as a thermal current. In general, for homogeneous systems, we can consider arbitrary moments of the single-particle distribution function:

$$
\mathrm{P}_{n}=\sum_{p} \hbar p_{z}\left(\beta E_{p}\right)^{n / 2} \mathrm{a}_{p}^{\dagger} \mathrm{a}_{p}
$$

as set of relevant observables $\left\{\mathrm{B}_{n}\right\}$. It can be shown that with increasing the number of moments, the result:

$$
\sigma=s \frac{\left.\left(k_{\mathrm{B}}\right)^{3 / 2}\left(4 \pi \epsilon_{0}\right)\right)^{2}}{m_{e}^{1 / 2} e^{2}} \frac{1}{\Lambda\left(p_{\text {therm }}\right)}
$$

(cf. Equation (29)) is improved, as can be shown with the Kohler variational principle; see $[13,16]$. The value $s=3 /(4 \sqrt{2 \pi})$ obtained from the single-moment approach is increasing to the limiting value $s=2^{5 / 2} / \pi^{3 / 2}$. For details, see $[4,13,14]$, where also other thermoelectric effects in plasmas are considered.

Single-particle distribution function and the general form of the linearized Boltzmann equation. Kinetic equations are obtained if the occupation numbers $\mathrm{n}_{p}$ of single-(quasi-) particle states $|p\rangle$ are taken as the set of relevant observables $\left\{\mathrm{B}_{n}\right\}$. In thermal equilibrium, neglecting interactions, the averaged occupation numbers of the single-electron states are given by the Fermi distribution function (14), $\left\langle\mathrm{n}_{p}\right\rangle_{\mathrm{eq}}=\operatorname{Tr}\left\{\rho_{\mathrm{eq}} \mathrm{n}_{p}\right\}=f_{1}^{0}\left(E_{p}\right)$. We consider the fluctuations of the occupation numbers $\Delta \mathrm{n}_{p}=\mathrm{n}_{p}-f_{1}^{0}\left(E_{p}\right)$ as relevant observables. The response equations, which eliminate the corresponding response parameters $F_{p}(t)$, have the structure of a linear system of coupled Boltzmann equations for the quasiparticles (see [16]):

$$
\frac{e}{m_{e}} \boldsymbol{E}_{\mathrm{ext}} \cdot\left[\left(\mathbf{P} \mid \mathrm{n}_{p}\right)+\left\langle\mathbf{P} ; \dot{\mathrm{n}}_{p}\right\rangle_{i \epsilon}\right]=\sum_{p^{\prime}} F_{p^{\prime}} P_{p^{\prime} p}
$$

with $P_{p^{\prime} p}=\left(\dot{\mathrm{n}}_{p^{\prime}} \mid \Delta \mathrm{n}_{p}\right)+\left\langle\dot{\mathrm{n}}_{p^{\prime}} ; \dot{\mathrm{n}}_{p}\right\rangle_{\mathrm{i} \epsilon}$. The response parameters $F_{p}(t)$ are related to the averaged occupation numbers as:

$$
f_{1}(p)=\operatorname{Tr}\left\{\rho(t) \mathrm{n}_{p}\right\}=f_{1}^{0}\left(E_{p}\right)+\beta \sum_{p^{\prime}} F_{p^{\prime}}\left(\Delta \mathrm{n}_{p^{\prime}} \mid \Delta \mathrm{n}_{p}\right) .
$$


The general form of the linear Boltzmann equation (71) can be compared with the expression obtained from kinetic theory. The left-hand side can be interpreted as the drift term, where self-energy effects are included in the correlation function $\left\langle\mathbf{P} ; \dot{\mathrm{n}}_{p}\right\rangle_{\mathrm{i}}$. The collision operator is given by $\left\langle\dot{\mathrm{n}}_{p^{\prime}} ; \dot{\mathrm{n}}_{p}\right\rangle_{\mathrm{i} \epsilon}$. Because the operators $\mathrm{n}_{p}$ are commuting, from the Kubo identity, it follows $\left(\dot{\mathrm{n}}_{p^{\prime}} \mid \mathrm{n}_{p}\right)=(1 / \hbar \beta)\left\langle\left[\mathrm{n}_{p^{\prime}}, \mathrm{n}_{p}\right]\right\rangle=0$. More precisely, the collision operator can be expressed in terms of the correlation function of the stochastic part of fluctuations; cf. Equation (67). The further evaluation of the conductivity is according to the kinetic approach in Section 3.

Two-particle distribution function, bound states. The question arises whether the conductivity, in particular the virial coefficient $A(T)$ (29) for the Lorentz plasma, is modified if the set of relevant observables is further extended. In the next step, we can consider the non-equilibrium two-particle distributions; see $[4,11]$. However, the corresponding corrections appear only in higher orders of the virial expansion (29). It seems that the virial coefficient $A(T)$ is an exact result. However, it is not clear whether in higher orders of density, singularities appear that can modify this result after partial summation of singular terms.

Another interesting quantum phenomenon is the formation of bound states. Such two-particle correlations can also be used to extend the set of relevant observables [22,23]. In particular, in low-density plasmas, such correlations are difficult to form dynamically and need a long relaxation time because a third particle is needed to fulfill the conservation laws. However, at fixed $T$, the concentration of bound states becomes small in the low density limit according to the mass action law.

We considered the interaction with uncorrelated ions, with structure factor $S(\boldsymbol{q})=1$. Multiple scattering by ordered ions with structure factor $S(\boldsymbol{q}) \neq 1$ will modify the result (27). In particular, for a perfect lattice, the electron system is described by Bloch states forming a band structure, and scattering disappears so that the conductivity becomes infinite (64).

Here, a main problem emerges. The electron Hamiltonian of the adiabatic Lorentz model (4) is bilinear and can, in principle, be diagonalized. We obtain stationary states as the exact solution, and the question arises from where dissipation in the system is coming. If an initial state is prepared with definite momentum by superposition of such exact solutions, the scattering into different directions of momentum is similar to the spreading out of a wave packet, and dissipation is only possible if the coherence is destroyed. The scattering by ions changes the total momentum of the electron system, but this cannot be considered as a dissipative process. In addition, for a closed circuit discussed above, we do not have asymptotically-free momentum states. Nevertheless, dissipation happens in real systems.

\section{Open Systems}

Flow of energy. We presented a nice and consistent approach to the electrical conductivity. The generalized linear response theory reproduces not only the low-density limit, which is also correctly described by kinetic theory, but gives the opportunity to treat also dense charged particle systems. However, the flow of energy is not correctly described. Because this is of second order in the external field, which determines the deviation from equilibrium, the account of the energy flow will not modify the results obtained in linear response theory.

Let us consider the system energy $\mathrm{H}_{S}$ (4). The dynamics is described by the Hamiltonian $\mathrm{H}^{t}=\mathrm{H}_{\mathrm{S}}+\mathrm{H}_{\mathrm{F}}^{t}$, which includes the field $E_{\text {ext }}(t)$. Using the extended von Neumann equation, we calculate the change of the energy of the electron system:

$$
\frac{\mathrm{d}}{\mathrm{d} t} \operatorname{Tr}\left\{\rho(t) \mathrm{H}_{\mathrm{S}}\right\}=\operatorname{Tr}\left\{\rho(t) \frac{\mathrm{i}}{\hbar}\left[\mathrm{H}_{\mathrm{F}}^{t}, \mathrm{H}_{\mathrm{S}}\right]\right\}-\epsilon \operatorname{Tr}\left\{\left[\rho(t)-\rho_{\text {rel }}(t)\right] \mathrm{H}_{\mathrm{S}}\right\} .
$$

We immediately see the import of electrical power $\Omega_{0} j \cdot \boldsymbol{E}_{\text {ext }}$ from the first term on the right-hand side. The second term becomes zero for $\epsilon \rightarrow 0$. Consequently, the average system energy is increasing with time. 
This is in contradiction to the demand of a stationary, homogeneous solution with constant current density $j$. Electrons that enter the open system have the same properties (average particle density, average current density, average energy density, temperature) as electrons that leave the system, as demanded by homogeneity. As discussed earlier, this contradiction is solved if the coupling to a bath is taken into account. Then, the dynamical evolution of the system according to $\mathrm{H}^{t}=\mathrm{H}_{\mathrm{S}}+\mathrm{H}_{\mathrm{F}}^{t}$ is not complete, and the influence of the bath is missing. We have to treat an open system where energy can be exported by coupling to a bath. We expect that the details of this coupling are not relevant for the calculation of the conductivity. However, the bath coupling is of relevance for the production of entropy. We discuss here the coupling to a system of harmonic oscillators as realized by phonons or photons. In the subsequent section, we show how the Zubarev method of the NSO may be modified to include the effects of the bath.

Harmonic-oscillator bath. The system of harmonic oscillators describing the excitations with wave vector $\boldsymbol{q}$ and polarization $\boldsymbol{e}_{q}$ is given by the Hamiltonian:

$$
\mathrm{H}_{\text {h.o. }}=\sum_{q} \omega_{q} \mathrm{~b}_{q}^{\dagger} \mathrm{b}_{q} .
$$

As the dispersion relation, we take $\omega_{q}=c|q|$ with $c$ as the velocity of light for the photon system or as the velocity of sound for acoustic phonons. For the interaction of the electron system with the phonon bath, the standard Froehlich expression:

$$
\mathrm{H}_{\text {int }}=\frac{\mathrm{i} c_{\mathrm{ep}}}{\Omega_{0}^{1 / 2}} \sum_{p, q} \sqrt{q} \mathrm{a}_{p+q}^{\dagger} \mathrm{a}_{p}\left(\mathrm{~b}_{q}-\mathrm{b}_{q}^{\dagger}\right)
$$

can be taken, with the electron-phonon coupling parameter $c_{\mathrm{ep}} \propto M_{i}^{-1 / 2}$. Note that we can also treat the electron-phonon interaction as a process to produce electrical conductivity [19].

Photon bath. In this work, we focus on the electron-photon interaction $\mathrm{H}_{\mathrm{int}}=e \sum_{i}^{N_{e}} \mathbf{r}_{\mathbf{i}} \cdot \mathbf{E}$. In contrast to Equation (5) where $E_{\text {ext }}(\boldsymbol{r}, t)$ denotes an external field, $\mathbf{E}(\boldsymbol{r}, t)$ is the operator of the fluctuating Maxwell field. In Fourier space, in the long-wavelength limit, we have the dipole approximation, $\mathrm{H}_{\text {int }}=e \mathbf{R} \cdot \mathbf{E}(\boldsymbol{q}=0)$. The system of harmonic oscillators is strongly coupled to a thermal bath so that the temperature $T_{\text {ext }}$ is fixed. The NSO can be investigated [4] with the selection of relevant observables as degrees of freedom of the system, and the remaining (irrelevant) degrees of freedom define the bath. The relevant statistical operator is chosen as the direct product of the thermodynamic equilibrium for the phonon/photon system, $\rho_{\text {bath }}$, fixed by the external temperature $T_{\text {ext }}$, and the reduced system statistical operator obtained after tracing out the irrelevant degrees of freedom. Performing the Born-Markov and rotating-wave approximation, the quantum master equation is obtained,

$$
\begin{aligned}
&\left.\frac{\partial \rho_{\epsilon}(t)}{\partial t}+\frac{\mathrm{i}}{\hbar}\left[\mathrm{H}^{t}, \rho_{\epsilon}(t)\right]=-\frac{1}{\hbar^{2}} \int_{-\infty}^{0} \mathrm{~d} \tau \mathrm{e}^{-\epsilon \tau} \stackrel{[}{[} \mathrm{H}_{\text {int }} \mathrm{H}_{\text {int }}(\tau)\right\rangle_{\text {bath }} \rho_{\epsilon}(t)+\rho_{\epsilon}(t)\left\langle\mathrm{H}_{\text {int }}(\tau) \mathrm{H}_{\text {int }}\right\rangle_{\text {bath }} \\
&\left.-\operatorname{Tr}_{\text {bath }}\left\{\mathrm{H}_{\text {int }} \rho_{\epsilon}(t) \rho_{\text {bath }} \mathrm{H}_{\text {int }}(\tau)+\mathrm{H}_{\text {int }}(\tau) \rho_{\epsilon}(t) \rho_{\text {bath }} H_{\text {int }}\right\}\right] .
\end{aligned}
$$

The notation $\langle\ldots\rangle_{\text {bath }}$ means average with respect to the phonon/photon bath, $\rho_{\text {bath }}=Z_{\text {bath }}^{-1} \mathrm{e}^{-\mathrm{H}_{\text {h.o. }} / k_{\mathrm{B}} T_{\text {ext }}}$. A further thermostat is needed to ensure thermodynamic equilibrium with temperature $T_{\text {ext }}$ [4]. The evaluation of the right-hand side is given below, Equation (82).

Electromagnetic field. The evaluation of the field averages with the harmonic-oscillator bath $\rho_{\text {bath }}$ can be performed. Finally, we give the result for the blackbody radiation $[4,24]$ with the field $\mathbf{E}(\boldsymbol{r}, t)$ :

$$
\Gamma_{i j}(\omega)=\int_{0}^{\infty} \mathrm{d} \tau \mathrm{e}^{\mathrm{i}(\omega+\mathrm{i} \varepsilon) \tau}\left\langle E_{i}(\tau) E_{j}(0)\right\rangle_{\text {bath }}=\delta_{i j}\left(\frac{1}{2} \gamma(\omega)+\mathrm{i} S(\omega)\right)
$$


with $\gamma(\omega)=4 \omega^{3}\left[1+n_{\mathrm{B}}(\omega)\right] /\left(3 \hbar c^{3}\right)$, and the principal value expression and:

$$
S(\omega)=\frac{2}{3 \pi \hbar c^{3}} \mathcal{P} \int_{0}^{\infty} \mathrm{d} \omega_{q} \omega_{q}^{3}\left[\frac{1+n_{\mathrm{B}}\left(\omega_{q}\right)}{\omega-\omega_{q}}+\frac{n_{\mathrm{B}}\left(\omega_{q}\right)}{\omega+\omega_{q}}\right] .
$$

Note that the Planck distribution (10) satisfies $n_{\mathrm{B}}(-\omega)=-\left[1+n_{\mathrm{B}}(\omega)\right]$ such that $\gamma(\omega)=4 \omega^{3}[1+$ $\left.n_{\mathrm{B}}(\omega)\right] /\left(3 \hbar c^{3}\right)$ for $\omega>0$ and $\gamma(\omega)=4|\omega|^{3} n_{\mathrm{B}}(|\omega|) /\left(3 \hbar c^{3}\right)$ for $\omega<0$.

The resulting quantum master equation describing the coupling such as atoms to the radiation field in dipole approximation, $\mathrm{H}_{\mathrm{int}}=-e \mathbf{R} \cdot \mathbf{E}$,

$$
\frac{\partial}{\partial t} \rho_{\epsilon}(t)-\frac{1}{\mathrm{i} \hbar}\left[\mathrm{H}_{S}, \rho_{\epsilon}(t)\right]-\frac{1}{\mathrm{i} \hbar}\left[\mathrm{H}_{\mathrm{infl}}, \rho_{\epsilon}(t)\right]=\mathcal{D}^{\prime}\left[\rho_{\epsilon}(t)\right] .
$$

has the Lindblad form. We perform the spectral decomposition with respect to the (discrete) eigenstates $\left|\phi_{n}\right\rangle$ of $\mathrm{H}_{\mathrm{S}}$

$$
\begin{aligned}
\mathbf{R}(\omega) & =\int_{-\infty}^{\infty} \mathrm{d} t \mathrm{e}^{\mathrm{i} \omega\left(t-t_{0}\right)} \mathrm{e}^{\mathrm{i} \mathrm{H}_{S}\left(t-t_{0}\right) / \hbar} \mathbf{R e}^{-\mathrm{iH}_{S}\left(t-t_{0}\right) / \hbar}=\mathbf{R}^{\dagger}(-\omega) \\
& =2 \pi \hbar \sum_{n, m} \delta\left(E_{S, n}-E_{\mathrm{S}, m}+\hbar \omega\right)\left|\phi_{n}\right\rangle\left\langle\phi_{n}|\mathbf{R}| \phi_{m}\right\rangle\left\langle\phi_{m}\right|
\end{aligned}
$$

The influence Hamiltonian:

$$
\mathrm{H}_{\mathrm{infl}}=e^{2} \hbar \int \mathrm{d} \omega S(\omega) \mathbf{R}^{\dagger}(\omega) \cdot \mathbf{R}(\omega)
$$

leads to a renormalization of the system Hamiltonian $\mathrm{H}_{\mathrm{S}}$ that is induced by the vacuum fluctuations of the radiation field (Lamb shift) and by the thermally-induced processes (Stark shift). The dissipator of the quantum master equation reads:

$$
\begin{aligned}
\mathcal{D}^{\prime}\left[\rho_{\epsilon}(t)\right]= & \int_{0}^{\infty} \mathrm{d} \omega \frac{4 e^{2} \omega^{3}}{3 \hbar c^{3}}\left[1+n_{\mathrm{B}}(\omega)\right]\left[\mathbf{R}(\omega) \rho_{\epsilon}(t) \mathbf{R}^{\dagger}(\omega)-\frac{1}{2}\left\{\mathbf{R}^{\dagger}(\omega) \mathbf{R}(\omega), \rho_{\epsilon}(t)\right\}\right] \\
& +\int_{0}^{\infty} \mathrm{d} \omega \frac{4 e^{2} \omega^{3}}{3 \hbar c^{3}} n_{\mathrm{B}}(\omega)\left[\mathbf{R}^{\dagger}(\omega) \rho_{\epsilon}(t) \mathbf{R}(\omega)-\frac{1}{2}\left\{\mathbf{R}(\omega) \mathbf{R}^{\dagger}(\omega), \rho_{\epsilon}(t)\right\}\right]
\end{aligned}
$$

where the integral over the negative frequencies has been transformed into positive frequencies. The influence term (81) is used to dress the electrons. Only the dissipator (82) is considered for the export of energy.

This result can be interpreted in a simple way. The application of the destruction operator $\mathbf{R}(\omega)$ on a state of the system lowers its energy by the amount $\hbar \omega$ and describes the emission of a photon. The transition rate $\frac{4 \omega^{3}}{3 \hbar c^{3}}\left[1+n_{\mathrm{B}}(\omega)\right]$ contains the spontaneous emission, as well as the thermal emission of photons. The term $\mathbf{R}^{\dagger}(\omega)$ gives the creation of photons with transition rate $\frac{4 \omega^{3}}{3 \hbar c^{3}} n_{\mathrm{B}}(\omega)$ describing the absorption of photons.

\section{The Relaxation Term}

Dissipator and relaxation. We can introduce the coupling to the thermal bath in different ways. After we described it by a Lindblad operator (79), based on a detailed description of interaction processes and performing some approximations, we now discuss whether we can also describe the influence of the bath by a relaxation term that describes the influence of a bath in a global, macroscopic way. The use of a relaxation time is very common in nonequilibrium statistical physics; see Section 3. We introduce the relaxation time as a characteristic, semiempirical quantity, which may be derived 
from a microscopic consideration. We analyze the von Neumann equation with a relaxation term similar to the source term (55),

$$
\frac{\partial}{\partial t} \rho_{\eta}(t)-\frac{1}{\mathrm{i} \hbar}\left[\left(\mathrm{H}_{\mathrm{S}}+\mathrm{H}_{\mathrm{F}}^{t}\right), \rho_{\eta}(t)\right]=-\eta\left[\rho_{\eta}(t)-\rho_{\mathrm{rel}}(t)\right] .
$$

Now, the relaxation parameter (superoperator) $\eta$ is finite and models the influence of the bath. The relevant statistical operator $\rho_{\text {rel }}(t)$ is considered no longer as the memory of the known averages in the past, to realize the initial, causal conditions. It describes the goal to which the evolution goes, a teleological distribution. It is determined by the external conditions as discussed below.

The idea is that the irrelevant degrees of freedom are strongly relaxed to a quasi-equilibrium, similar to the Enskog approach to solve the Boltzmann equation. The influence on the average motion of the relevant observables is replaced by a transport coefficient similar to the friction force in the Langevin equation. A more detailed description will relate this macroscopic friction force to the correlation function of stochastic forces, as known from the Brownian motion.

Ideal gas with friction. Let us consider a simple example for illustration. We have relevant (the electron variables) and irrelevant (ionic) observables. As discussed above, the relevant part of the dynamics is described explicitly, whereas the irrelevant one is described globally, e.g., by a relaxation term. In our simple example, the relevant part of the dynamics is $\mathrm{H}_{S, \text { id }}=\sum_{i} \hbar^{2} p_{i}^{2} / 2 m_{e}$. We disregard the $e-i$ interaction, but introduce a friction term $\eta_{\mathrm{ei}}$, of course not as $\frac{\partial}{\partial t} \rho_{\eta}(t)=-\eta_{\mathrm{ei}} \rho_{\eta}(t)$, but, to conserve normalization, energy, and particle number, we can consider the relaxation term:

$$
\frac{\partial}{\partial t} \rho_{\eta}(t)-\frac{1}{\mathrm{i} \hbar}\left[\left(\mathrm{H}_{\mathrm{S}, \mathrm{id}}+\mathrm{H}_{\mathrm{F}}^{t}\right), \rho_{\eta}(t)\right]=-\eta_{\mathrm{ei}}\left[\rho_{\eta}(t)-\rho_{\mathrm{eq}, \mathrm{id}}\right],
$$

with the equilibrium distribution $\rho_{\text {eq,id }}=\exp \left[-\beta \mathrm{H}_{S, \text { id }}+\beta \mu_{e} N_{e}\right] / Z_{\text {eq,id }}$ defined by temperature, chemical potential, zero mean velocity, and the ideal gas Hamiltonian $\mathrm{H}_{\mathrm{S}, \mathrm{id}}=\sum_{p} E_{p} \mathrm{a}_{p}^{\dagger} \mathrm{a}_{p}$ (Note that $\mathrm{H}_{\mathrm{F}}^{t}$ is not included. We demand a homogeneous distribution, and the external field would be compensated by a position-dependent chemical potential.). The norm and particle number are conserved, and for the average momentum, we have:

$$
\frac{\mathrm{d}}{\mathrm{d} t}\langle\mathrm{P}\rangle_{\eta}^{t}=\operatorname{Tr}\left\{\frac{\partial}{\partial t} \rho_{\eta}(t) \mathrm{P}\right\}=-e E_{\mathrm{ext}} N_{e}-\eta_{\mathrm{ei}}\langle\mathrm{P}\rangle_{\eta}^{t}
$$

with the stationary result $j=\frac{-e}{m_{e} \Omega_{0}}\langle\mathrm{P}\rangle_{\text {stat }}=\frac{e^{2} n_{e}}{m_{e} \eta_{\mathrm{ei}}} E_{\mathrm{ext}}$ so that $\sigma=n_{e} e^{2} /\left(m_{e} \eta_{\mathrm{ei}}\right)$ results; see Equation (64). Comparing to the microscopic calculation (28), we identify $\eta_{\mathrm{ei}}=1 / \bar{\tau}^{\text {transp }}$.

Dynamical collision frequency. We have shown that the phenomenological relaxation time $1 / \eta_{\mathrm{ei}}$ is related to the microscopic $e-i$ interaction solved in Section 3. We know from the Langevin equation that transport (friction) coefficients are related to the correlation function of stochastic forces. We have to consider a stochastic process, and stochastic forces are needed to maintain thermal irregular motion, cf. Equation (67). We demonstrate this relation for the ideal gas with friction to describe the collisions with ions.

Within the Zubarev NSO approach, we derive the response of the system to a time-dependent external field; see $[14,20]$. Medium modifications of electromagnetic fields in an isotropic plasma are described by the dielectric permittivity tensor $\hat{\varepsilon}(q, \omega)$. In the long-wavelength limit $q \rightarrow 0$ (relevant for the emission and absorption of visible light considered here), the transverse and longitudinal dielectric function are identical. The dielectric function is written in the generalized Drude-like form with $\omega_{\mathrm{pl}}^{2}=n_{e} e^{2} /\left(\epsilon_{0} m_{e}\right)$,

$$
\varepsilon(q \rightarrow 0, \omega)=1=1+\frac{\mathrm{i}}{\epsilon_{0} \omega} \sigma(q \rightarrow 0, \omega)=1-\frac{\omega_{\mathrm{pl}}^{2}}{\omega[\omega+\mathrm{i} v(\omega)]} .
$$


The dynamical collision frequency $v(\omega)$ is obtained from the frequency-dependent force-force correlation function; see [25] and Equations (67) and (68).

$$
v(\omega)=\frac{\beta}{N_{e} m_{e}}\langle\dot{\mathrm{P}} ; \dot{\mathrm{P}}\rangle_{\omega+\mathrm{i} \epsilon} .
$$

For the DC conductivity follows $\sigma=\epsilon_{0} \omega_{\mathrm{pl}}^{2} / \nu(0)=n_{e} e^{2} /\left[m_{e} v(0)\right]$, so that we identify $\eta_{\mathrm{ei}}=v(0)$.

Note that this global description of the effect of $e-i$ interaction by a relaxation term is very crude, but was successfully applied to derive the Mermin dielectric function $\varepsilon^{\operatorname{Mermin}}(q, \omega)$ [26]. The contribution of $e-i$ interaction for $q=0$ is described by the dynamical collision frequency $v(\omega)$. The extension to finite $q$ was possible taking the conservation laws for particle number, etc., into account.

Relaxation of internal energy, ideal gas. The relaxation of the electron momentum owing to $e-i$ collisions is correctly described. The conservation of the norm and particle number is realized by construction. For the internal energy $U_{S, \text { id }}(t)=\left\langle\mathrm{H}_{S, \mathrm{id}}\right\rangle_{\eta}^{t}$, we have with Equation (84):

$$
\frac{\mathrm{d}}{\mathrm{d} t} U_{\mathrm{S}, \mathrm{id}}(t)=j E_{\mathrm{ext}} \Omega_{0}-\eta_{\text {bath }}\left[U_{\mathrm{S}, \mathrm{id}}(t)-\frac{3}{2} k_{\mathrm{B}} T_{\text {ext }} N_{e}\right]
$$

with the stationary result $U_{\text {stat }}=\frac{3}{2} k_{\mathrm{B}} T_{\text {ext }} N_{e}+j E_{\text {ext }} \Omega_{0} / \eta_{\text {bath }}$. The values $j_{\text {eq }}=0$ and $U_{\text {eq }}=\frac{3}{2} k_{\mathrm{B}} T_{\text {ext }} N_{e}$ as demanded by the bath are not reached in the stationary state.

The relaxation coefficient $\eta_{\text {bath }}$ is different from $\eta_{\mathrm{ei}}$ because elastic $e-i$ collisions, which are relevant for the relaxation of momentum, will not contribute to the relaxation of internal energy. Other phenomena like bremsstrahlung emission (see Section 8) give the microscopic process for this relaxation term. The relaxation parameter $\eta$ should be considered as the superoperator in the space of the dynamical variables $\left\{\mathrm{B}_{n}\right\}$ of the system, similar to the relaxation time $1 / \tau^{\text {transp }}\left(E_{p}\right)(24)$ acting on states $|p\rangle$.

Global energy relaxation. As a simple example for energy relaxation, we discuss temperature relaxation for a system in local thermodynamic equilibrium. For the ideal classical gas of electrons, we have the equilibrium distribution (see Equation (14)):

$$
f_{1}^{0}\left(E_{p}\right)=\frac{n_{e}}{2}\left(\frac{2 \pi \hbar^{2}}{m_{e} k_{\mathrm{B}} T}\right)^{3 / 2} \mathrm{e}^{-\frac{\hbar^{2} p^{2}}{2 m_{e} k_{\mathrm{B}} T}} .
$$

The change $T \rightarrow T_{\text {ext }}$ where $T_{\text {ext }}$ is the temperature of the bath can be described by the map:

$$
f_{1}^{0}\left(E_{p}\right)=f_{1}^{0}\left(\frac{\hbar^{2} p^{2}}{2 m_{e}}\right) \rightarrow\left(\frac{T}{T_{\mathrm{ext}}}\right)^{3 / 2} f_{1}^{0}\left(\frac{\hbar^{2} p^{2} T_{\mathrm{ext}}}{2 m_{e} T}\right) .
$$

The equation of evolution for $T(t)$ is obtained from:

$$
\frac{\partial}{\partial t} f_{1}^{0}\left(E_{p} ; T(t)\right)=-\eta_{\text {bath }}\left[f_{1}^{0}\left(E_{p} ; T(t)\right)-f_{1}^{0}\left(E_{p} ; T_{\text {ext }}\right)\right]
$$

so that near $T \approx T_{\text {ext }}$, we find $\dot{T}(t)=-\eta_{\text {bath }}\left[T(t)-T_{\text {ext }}\right]$. This global relaxation of $T$ scales all momenta in the same way and can be replaced by a more detailed description within a microscopic approach.

Hamiltonian dynamics. Now, we discuss the NSO method presented in Section 4. The dynamical evolution owing to the $e-i$ interaction, given by $\mathrm{H}_{\mathrm{S}}$, is treated microscopically so that it must be taken 
off the relaxation term. However, this microscopic description is not complete because the interaction with a bath is not included. We obtain from Equation (56):

$$
\left.\frac{\mathrm{d}}{\mathrm{d} t}\left\langle\mathrm{~B}_{n}\right\rangle^{t}=\frac{\mathrm{i}}{\hbar} \operatorname{Tr}\left\{\left[\mathrm{H}^{t}, \mathrm{~B}_{n}\right] \rho_{\eta}(t)\right]\right\}-\eta_{\text {bath }}\left[\operatorname{Tr}\left\{\rho_{\eta}(t) \mathrm{B}_{n}\right\}-\operatorname{Tr}\left\{\rho_{\text {rel }}(t) \mathrm{B}_{n}\right\}\right] .
$$

Now, the relaxation coefficient $\eta_{\text {bath }}$ only describes the interaction with the bath. It is clearly seen that this relation becomes wrong for $\lim \eta_{\text {bath }} \rightarrow 0$ if the dynamics $\mathrm{H}^{t}=\mathrm{H}_{\mathrm{S}}+\mathrm{H}_{\mathrm{F}}^{t}$ is incomplete, i.e., not containing the coupling to the bath, and $\left\langle\mathrm{B}_{n}\right\rangle^{t}$ is a prescribed time evolution, which respects the influence of the bath. In particular, in a stationary state, the averages of the dynamical observables of the system, including the internal energy $\left\langle\mathrm{H}_{\mathrm{S}}\right\rangle^{t}$, should not depend on time. The appropriate choice of the source term, in particular the relevant operator $\rho_{\text {rel }}(t)$, can be performed so that double counting of the Hamiltonian dynamics $\mathrm{H}^{t}$ is avoided.

As discussed above for an isolated, closed system, the source term can be constructed such that the dynamical evolution is projected out. The condition (43):

$$
\left\langle\mathrm{B}_{n}\right\rangle^{t}=\left\langle\mathrm{B}_{n}\right\rangle_{\text {rel }}^{t}
$$

makes the time evolution (56) of the relevant observables $\mathrm{B}_{n}$ purely dynamical, i.e., according to $\mathrm{H}^{t}$, and independent of the value of $\epsilon$. However, in open systems, the dynamical observables of the system may also be influenced by the bath, so that the Hamiltonian time evolution, neglecting the influence of the bath, is incomplete, and therefore, it is in conflict with the demanded self-consistency condition (93).

Maximum production of entropy. We propose another definition of the relevant statistical operator for open systems in the stationary state. Given properties are only a small number of control observables. In our case, the relevant operator is characterized by the density $n_{e}$, the current density $j$ controlled by the external field $E_{\text {ext }}$, and the internal energy density controlled by the bath temperature $T_{\text {ext }}$. The mean values of further observables are not measured, so that Equation (93) is meaningless. Instead, an arbitrary number of dynamical observables $\left\{B_{n}\right\}$ of the system may be considered, and their averages are determined by the Kohler variational principle [16], where the arbitrary time dependence of the external field is considered. It can be related to the principle of the extremum of entropy production given by Prigogine and Glansdorff [1]. The static case $\omega=0$ has been considered in $[4,14]$.

Coming back to our example of DC conductivity, some control parameters are known in the past $t_{1}<t$ : the volume $\Omega_{0}$, the particle density $n_{e}$, the temperature $T_{\text {ext }}$, and the external field $\mathbf{E}_{\text {ext }}$, which, in general, may depend on time $t_{1}$. A Gibbs ensemble $\rho_{\text {eq }}$ with $\mathrm{H}_{\mathrm{S}}$ (remember that $\mathrm{H}_{\mathrm{F}}^{t}$ is compensated by the chemical potential to have homogeneous solution), and the external conditions $\Omega_{0}, n_{e}, T_{\text {ext }}$ are compatible with the demanded properties, but not very appropriate to describe the stationary state; see the discussion of the Kubo formula (63). At least, we expect to have a stationary distribution with a finite average momentum current density $j=\left(-e \hbar / m_{e} \Omega_{0}\right)\langle\mathbf{P}\rangle$, which characterizes the relevant distribution. We can add further moments or the detailed single-particle distribution function $f_{1}(\boldsymbol{p}, t)$ as the set $\left\{\mathrm{B}_{n}\right\}$ of relevant observables. The averages of these relevant observables are not prescribed by the self-consistency relations (93), but by the maximum production of information entropy, as shown, e.g., by the Kohler variational principle. The principle of maximum information entropy fixes all remaining (irrelevant) observables, such as missing moments if only a finite number of moments is taken, or two-particle correlation functions. The construction of the relevant statistical operator $\rho_{\text {rel }}(t)$ has to be considered as a variational problem. According to the Kohler variational principle of the maximum production of relevant entropy at a given external field $E_{\text {ext }}$, we find that the more relevant observables $\left\{\mathrm{B}_{n}\right\}$ are included, the better (larger) being the result for the conductivity; see the examples in Section 5.

The approach presented here is known as synchronization or the sequence of different stages of non-equilibrium. Similar to the Enskog equation, we assume strong equilibration of the higher 
correlation functions (collision time scale), followed by the equilibration of the single-particle distribution function (free flight time scale) and the position-dependent hydrodynamic scale (transport and diffusion). The higher correlations are already equilibrated. For the conductivity in the low-density limit, the single-particle distribution function is sufficient. Higher order distribution functions relax quickly. An important example is the dressing of free particles to become quasiparticles with self-energy according to local thermodynamic equilibrium. We can discuss this as a fast synchronization of the irrelevant observables to the quasi-equilibrium, relevant distribution.

Dynamics and relaxation. It is our main issue to construct the equation of evolution with a relaxation term avoiding double counting of the dynamical part $\mathrm{H}^{t}$ of the interaction. For our open system, the time evolution operator $\mathrm{U}\left(t, t_{0}\right)$, Equation (49), has to be completed to contain also the interaction with the bath. In the expressions (58) and (59), the interaction with the bath has to be added. Within a microscopic approach, $\mathrm{d}\left\langle\mathrm{B}_{n}\right\rangle^{t} / \mathrm{d} t$ should also have the contribution of interaction with the bath. As is well known, a dissipator (76) can be derived so that $\rho(t)$ follows from a quantum master equation. Because of this additional interaction with the bath, the self-consistency condition (43) and (93) becomes obsolete for the averages of the relevant observables. Instead, only the external conditions $E_{\text {ext }}, T_{\text {ext }}$ are given, and the averages of the relevant observables $\left\{B_{n}\right\}$ are determined from the maximum production of the relevant entropy.

As before, we approximately introduce a relevant statistical operator $\rho_{\text {rel }}$, which is optimized with respect to the given dynamics $\mathrm{H}_{S}$ of the system, i.e., the maximum of production of the relevant entropy according to the Kohler variational principle. For this, we select out a set of relevant observables $\left\{B_{n}\right\}$ and find the corresponding Lagrange parameters $F_{n}$ solving the linear system $\sum_{n} P_{m n} F_{n}=D_{m} E_{\text {ext }}$ of Equation (65). This approximation can be improved taking into account the influence of the bath, for instance replacing $\epsilon$ by $\eta_{\text {bath }}$ calculating the correlation functions. Now, we solve the problem of the intrinsic energy $\left\langle\mathrm{H}_{\mathrm{S}}\right\rangle^{t}$, which should be constant in the stationary state. The von Neumann equation with the friction term (83) gives for the time derivative:

$$
0=j E_{\text {ext }}-\frac{3}{2} n_{e} \eta_{\text {bath }} k_{\mathrm{B}}\left(T-T_{\text {ext }}\right)
$$

so that the temperature in the system is $T=T_{\text {ext }}+\frac{2}{3 \eta_{\text {bath }} k_{\mathrm{B}}} \sigma E_{\text {ext }}^{2}$, cf. Equation (36). In linear response, the temperature difference $\Delta T=T-T_{\text {ext }} \propto E_{\text {ext }}^{2}$ can be neglected. To estimate $\Delta T$, we need a microscopic description of the bath coupling as discussed in Section 8.

\section{Microscopic Description of the Bath Coupling}

Dynamical collision frequency and bremsstrahlung emission. Bremsstrahlung emission has been considered as a possible process to export the energy from the system to the bath. It is related to absorption, which is obtained from classical field theory. Quantum field theory is needed to obtain spontaneous emission, cf. Equations (11)-(13). The dielectric function $\varepsilon(\omega)$ is connected to the index of refraction $n(\omega)$ and the absorption coefficient $\alpha(\omega)$ by $n(\omega)+(\mathrm{ic} / 2 \omega) \alpha(\omega)=\sqrt{\varepsilon(\omega)}$. The absorption coefficient is related to the bremsstrahlung radiation. In Born approximation, we obtain [14]:

$$
\operatorname{Re} v(\omega)=\frac{e^{4} n_{i}}{12 \pi^{2} \varepsilon_{0}^{2} m_{e}}\left(\frac{2 \pi m_{e}}{k_{\mathrm{B}} T}\right)^{1 / 2} \frac{1}{\hbar \omega}\left[e^{\beta \hbar \omega / 2}-e^{-\beta \hbar \omega / 2}\right] K_{0}\left(\frac{\hbar \omega}{2 k_{\mathrm{B}} T}\right)
$$

where $K_{0}(z)=\int_{0}^{\infty} \mathrm{d} t \cos (z \sinh t)$ is a modified Bessel function. The static ion structure factor is approximated as $S_{i}(q) \approx 1$. Comparing with the Kramers formula (13), the corresponding result for the Gaunt factor has been obtained. Further changes of Kramers' expression are obtained from the account of many-body effects.

As in the case of momentum relaxation described by the relaxation parameter $\eta_{\mathrm{e} i}$, we estimate the relaxation parameter $\eta_{\text {bath }}$ which describes the relaxation of internal energy (94). We consider the 
loss of energy of the electrons owing to bremsstrahlung emission, Equation (13). Integrating over $\omega$, the total emission power density is [27]:

$$
P\left[\mathrm{~W} / \mathrm{m}^{3}\right]=-\frac{1}{\Omega_{0}} \frac{\mathrm{d}}{\mathrm{d} t}\left\langle\mathrm{H}_{\mathrm{S}}\right\rangle=1.69 \times 10^{-38} \mathrm{Z}_{i}^{2} n_{e}\left[\mathrm{~m}^{-3}\right] n_{i}\left[\mathrm{~m}^{-3}\right]\left(k_{\mathrm{B}} T[\mathrm{eV}]\right)^{1 / 2} .
$$

This expression gives already the result in the corresponding SI units. For instance, typical experiments to measure the DC conductivity in dense plasmas [28] are performed at $k_{\mathrm{B}} T \approx 2 \mathrm{eV}$ and free electron density $n_{e} \approx 3 \times 10^{25} \mathrm{~m}^{-3}$. At electrical fields, $E_{\mathrm{ext}} \approx 100 \mathrm{~V} / \mathrm{m}$ linear behavior $j=\sigma E_{\text {ext }}$ has been observed, with $\sigma \approx 2 \times 10^{4}[\Omega \mathrm{m}]^{-1}$. Comparison with our results (29) has been performed in [11]. According to (96), the total emission power density is $P \approx 2 \times 10^{13} \mathrm{~W} / \mathrm{m}^{3}$. This loss of energy owing to bremsstrahlung radiation determines the cooling rate of the plasma and the temperature of the stationary state according to Equation (94). With $j E_{\mathrm{ext}}=\sigma E_{\mathrm{ext}}^{2} \approx 2 \times 10^{8} \mathrm{~W} / \mathrm{m}^{3}$, the temperature difference $\Delta T<1 \mathrm{~K}$ between the emitting system and the emitting bath is relatively small in the stationary case.

However, this estimation is valid only for a plasma that is optically thin, i.e., the radiation emitted by the electrons can escape from the plasma without reabsorption. In an optically-thick plasma, emitted radiation is reabsorbed (self-absorption) after a short distance compared to the size of the plasma. The balance of both emission and absorption processes in the stationary state leads to a lower efficiency of the energy transfer from the electrons to the radiation field and a corresponding higher plasma temperature in the stationary state. Then, the energy spectrum is constrained to the Planck spectrum (10). In particular, below the plasma frequency, electromagnetic radiation cannot propagate in the plasma. Radiation transport determines the export of energy, and the temperature of the radiation field $T_{\text {ext }}(\boldsymbol{r}, t)$ becomes dependent on the position. The constraining Planck spectrum in the low-frequency limit (Raleigh-Jeans law) can be used to define the temperature. According to the Stefan-Boltzmann law, the heat is related to temperature as a property of the Planck spectrum (10), which gives the occupation numbers of photon states, not showing the phase of the electromagnetic wave.

Quantum structure of electromagnetic fields. As in the case of momentum relaxation $\eta_{\mathrm{e} i}$, the energy relaxation $\eta_{\text {bath }}$ is related to a microscopic process, the interaction of the electrons with the radiation field. As known from the Brownian motion, behind the relaxation term, which describes the average motion, there is a stochastic process. This allows one to calculate the relaxation parameter $\eta_{\text {bath }}$ from the correlation function of stochastic forces (fluctuation-dissipation theorem); see Equation (67). Whereas for $\eta_{\mathrm{e}}$, the collision frequency $v(0)$ was considered, which describes the fluctuations of the Coulomb forces in the charged-particle system, we consider for $\eta_{\text {bath }}$ the vacuum fluctuations of the electromagnetic field. In particular, a quantum field theory is needed to describe spontaneous emission of radiation; for details, see, e.g., [4,24].

Let us consider the quantum fluctuations $\mathbf{E}(r, t), \mathbf{B}(r, t)$ in thermal equilibrium. The fluctuation properties of the electrical field are obtained from the Maxwell equations in free space. The vector potential in the Coulomb gauge leads to wave equations for the transverse vector potential, which may be solved by a plane wave decomposition with photon creation and annihilation operators $\mathrm{b}_{\lambda}(\boldsymbol{q})$, satisfying bosonic commutation relations. The commutator (where $[\mathrm{A}, \mathrm{B}]=\mathrm{AB}-\mathrm{BA}$ ) and the anticommutator (where $\{\mathrm{A}, \mathrm{B}\}=\mathrm{AB}+\mathrm{BA}$ ) function can be calculated; see [24]. As a result, for the anticommutator correlation function of the transverse electric field, averaged over the radiation field in thermal equilibrium at $T$, one obtains for the vector components with $x=\{c \Delta t, \Delta r\}$ :

$$
\begin{aligned}
& \left\langle\left\{E_{i}(x), E_{j}(0)\right\}\right\rangle=-\left(\delta_{i j}-\frac{x_{i} x_{j}}{r^{2}}\right) \frac{1}{2 \pi^{2} \tau_{T}^{3} r} \\
& \times\left[\frac{\cosh \left[(r / c-t) / \tau_{T}\right]}{\sinh ^{3}\left[(r / c-t) / \tau_{T}\right]}+\frac{\cosh \left[(r / c+t) / \tau_{T}\right]}{\sinh ^{3}\left[(r / c+t) / \tau_{T}\right]}\right]
\end{aligned}
$$


with $r=|\Delta r|, t=\Delta t$, and $\tau_{T}=\hbar /\left(\pi k_{\mathrm{B}} T\right)$ is the thermal correlation time. For $r \rightarrow 0$ follows, summing over $i, j$, the expression $\langle\{\mathbf{E}(0, t), \mathbf{E}(0)\}\rangle \approx \frac{6}{\pi^{2} t^{4}}$ for $|t| \ll \tau_{T}$. This corresponds to vacuum contribution $(T=0)$ and diverges near $t=0$.

The thermal contribution is:

$$
\langle\{\mathbf{E}(0, t), \mathbf{E}(0)\}\rangle_{\mathrm{th}}=\frac{1}{\pi^{2} \tau_{T}^{4}}\left[\frac{6+4 \sinh ^{2}\left(t / \tau_{T}\right)}{\sinh ^{4}\left(t / \tau_{T}\right)}-\frac{6}{\pi^{2}\left(t / \tau_{T}\right)^{4}}\right] .
$$

Performing $t \rightarrow 0$, we get the energy density of the field in thermal equilibrium as $u_{\text {th }}=$ $\langle\{\mathbf{E}(0), \mathbf{E}(0)\}\rangle_{\mathrm{th}} / 2=1 /\left(15 \pi^{2} \tau_{T}^{4}\right)$, which is the Stephan-Boltzmann law of black-body radiation. The long-time behavior $t \gg \tau_{T}$ results as $\langle\{\mathbf{E}(0, t), \mathbf{E}(0)\}\rangle_{\text {th }} \approx 16 /\left(\pi^{2} \tau_{T}^{4}\right) \exp \left(-2|t| / \tau_{T}\right)$. We can consider the local, but low-frequency limit of the spectral density (see, e.g., [4]):

$$
S_{E E}^{\text {therm }}(r=0, \omega)=\int \mathrm{d} t \mathrm{e}^{-\mathrm{i} \omega t}\langle\{\mathbf{E}(0, t), \mathbf{E}(0)\}\rangle_{\text {th }} \approx \frac{4}{\pi^{2} \tau_{T}^{3}} \frac{1}{1+\omega^{2} \tau_{T}^{2} / 4} .
$$

We also have:

$$
\Gamma(\omega)=1 / \hbar^{2} \int_{0}^{\infty} \mathrm{d} t \mathrm{e}^{-\mathrm{i}(\omega+\mathrm{i} \epsilon) t}\langle\{\mathbf{E}(0, t), \mathbf{E}(0)\}\rangle=\gamma(\omega) / 2+i S(\omega) .
$$

The thermal contribution has the low-frequency $\operatorname{limit}_{\lim } \operatorname{li0}_{E E}^{\text {therm }}(r=0, \omega)=4 /\left(\pi^{2} \tau_{T}^{3}\right)$, and the results give:

$$
\gamma \approx 4 \omega^{2} k_{\mathrm{B}} T /\left(3 \hbar c^{3}\right), \quad \lim _{\omega \rightarrow 0} S_{E E}^{\text {therm }}(\omega)=\frac{4 \zeta[3]}{3 \pi \hbar c^{3} \tau_{T}^{3}},
$$

with Apery's constant $\zeta[3]=1.20205$, cf. Equation (77). In addition to the vacuum fluctuations, we have thermal fluctuations of the electrical field proportional $T^{3}$. Considering this low-frequency limit, we can introduce a local $(r=0)$ temperature from the fluctuation spectrum of the electromagnetic fields.

Radiation damping. We cannot give here a detailed discussion of quantum electrodynamic processes. An interesting process is radiation damping. From classical electrodynamics, it is known that charged particles emit radiation if they are accelerated. Using the Larmor formula (12), the equation of motion for an electron that contains the interaction with the radiation field is the Abraham-Lorentz equation:

$$
m_{e} \dot{\boldsymbol{v}}(t)-\boldsymbol{F}_{\mathrm{ext}}(t)=\boldsymbol{F}_{\mathrm{rad}}(t)=\frac{e^{2}}{6 \pi \epsilon_{0} c^{3}} \ddot{\boldsymbol{v}}(t)=m_{e} \tau_{\mathrm{rad}} \ddot{\boldsymbol{v}}(t),
$$

where $\boldsymbol{F}_{\text {ext }}(t)$ denotes an external force. The radiation damping term is determined by the characteristic time $\tau_{\text {rad }}=e^{2} /\left(6 \pi \epsilon_{0} m_{e} c^{3}\right)=6.3 \times 10^{-24}$ s.

The Abraham-Lorentz Equation (102) can also be derived if the radiation degrees of freedom are eliminated, as discussed above in Section 6; see [24]. Different problems such as runaway solutions arise; see [4]. The interaction with the radiation field, in particular bremsstrahlung processes, leads to a loss of quantum coherence, to localization, and to the transition to classical behavior [24]. The bremsstrahlung is emitted during the collision of charged particles. Emission of photons can be considered as a measuring process to localize the charged particle during the collision process. A more detailed discussion of the suppression of quantum coherence can be found in [29]. The balance of emitted and absorbed power in the classical limit is (12):

$$
\frac{\mathrm{d}}{\mathrm{d} t}\left\langle\mathrm{H}_{\mathrm{S}}\right\rangle=-\frac{2 e^{2}}{3 \epsilon_{0} c^{3}} \sum_{i}^{N_{e}}\left(\frac{\mathrm{d}^{2} \boldsymbol{r}_{i}}{\mathrm{~d} t^{2}}\right)^{2}+e \sum_{i}^{N_{e}} \dot{\boldsymbol{r}}_{i} \cdot \boldsymbol{E}_{\mathrm{ext}}\left(\boldsymbol{r}_{i}, t\right),
$$


if reabsorption and coherence effects given by the ionic structure factor are discarded. The emission and absorption of radiation, as described by a quantum master equation, are some of the possibilities to solve the problem of the export of entropy.

\section{Conclusions}

von Neumann equation with the relaxation term. According to the Zubarev NSO method, a source term $-\epsilon\left[\rho(t)-\rho_{\text {rel }}(t)\right]$ was introduced into the von Neumann Equation (55), with lim $\epsilon \rightarrow 0$ after the thermodynamic limit. This source term is not a physical process, but rather a mathematical trick to select out the retarded solution of the equation of motion. Infinitesimal source terms to break a symmetry are known from other fields in physics such as phase transitions, for instance the direction of magnetization in the Heisenberg model or a phase in the superfluid phase, but also the Planck "Staubkorn" to establish the blackbody radiation spectrum in a "hohlraum". For a closed system with a known Hamiltonian, this source term has a remarkable property. We can select out an arbitrary set of relevant observables and construct the source term with the corresponding relevant statistical operator $\rho_{\text {rel }}(t)$. As a consequence of the self-consistency conditions (43) and (93), the dynamics of the relevant observables (56) obeys the Hamiltonian dynamics also for finite $\epsilon$. The invariance of the dynamics of the relevant observables with respect to the source term with arbitrary $\epsilon$ is a remarkable property of the extended von Neumann equation.

Open systems and relaxation term. We discussed open systems, in particular diabatic contact (exchange of energy, but not particles) with a thermal bath. The system is defined by the dynamical degrees of freedom and a Hamiltonian $\mathrm{H}^{t}=\mathrm{H}_{\mathrm{S}}+\mathrm{H}_{\mathrm{F}}^{t}$, which determines the equations of motion of the system, including the action of external fields $E_{\text {ext }}(t)$. In addition to the Hamiltonian dynamics, instead of the infinitesimal source term, a relaxation term with finite $\eta$ is introduced in Equation (83) to model the influence of the bath, e.g., prescribing the temperature $T_{\text {ext }}$. This finite source term is no longer interpreted as the initial conditions in the past to construct a solution of the von Neumann equation with time evolution $\mathrm{H}^{t}$, but to give a final state to which the distribution relaxes, at each time instant in the past. More generally, a final state is given by the external conditions, and the influence on the system, the coupling to a bath, is globally described by a relaxation term. The form of the relaxation term, in our case the relevant statistical operator $\rho_{\text {rel }}(t)$ and the phenomenological relaxation coefficient $\eta$, which, in general, is an operator, has to be chosen in an appropriate way. Note that alternative expressions for the relaxation term are possible. For instance, also for $\ln \rho(t)$, a von Neumann equation can be given, and a source term $-\epsilon\left[\ln \rho(t)-\ln \rho_{\text {rel }}(t)\right]$ and a corresponding relaxation term with $\epsilon \rightarrow \eta$ can be proposed.

The Bogoliubov principle of the weakening of initial correlations. According to the Bogoliubov principle of weakening of initial correlations, the missing correlations to get $\rho(t)$ are produced dynamically. However, this argument is not valid in the case of an open system considered here, if, in addition to the Hamiltonian dynamics, the coupling to the bath is taken into account. In contrast to the system described by a Hamiltonian, the dynamical evolution of the bath is not exactly known. The average with the NSO (53) will not give the empirical averages even if the relevant statistical operator $\rho_{\text {rel }}\left(t_{1}\right)$ is replaced by the exact $\rho\left(t_{1}\right)$. Only for a closed system with a known Hamiltonian, the Bogoliubov principle of the weakening of initial correlations is valid. For an open system, the missing correlations are not only produced by the Hamiltonian dynamics within the system, but are also influenced by the external, in general time-dependent conditions, which characterize the surroundings, the bath.

The self-consistency conditions. Let us consider an arbitrary, dynamical observable $\mathrm{B}_{n}$ of the system. If we know the history, i.e., $\left\langle\mathrm{B}_{n}\right\rangle^{t_{1}}$ in the past $t_{1}<t$, the correct time evolution from $t_{1}$ to $t$ should also contain the coupling to the bath. For example, this can be expressed in some approximation by a dissipator term (79). In this work, we propose to use a relaxation time approach to describe the external influence on the system. The time evolution of the average $\left\langle\mathrm{B}_{n}\right\rangle^{t}=\operatorname{Tr}\left\{\rho_{\eta}(t) \mathrm{B}_{n}\right\}$ is given by Equation (56) replacing $\epsilon \rightarrow \eta$. In general, the observables of the system are influenced by the bath; as an example, we considered the internal energy. Therefore, the relevant statistical operator $\rho_{\text {rel }}(t)$ is 
not determined by the self-consistency conditions (43) and (93), because then the influence of the bath on the time evolution of the average $\left\langle\mathrm{B}_{n}\right\rangle^{t}$ disappears. We have:

$$
\left\langle\mathrm{B}_{n}\right\rangle^{t}=\operatorname{Tr}\left\{\rho_{\eta}(t) \mathrm{B}_{n}\right\} \neq \operatorname{Tr}\left\{\rho_{\text {rel }}(t) \mathrm{B}_{n}\right\}=\left\langle\mathrm{B}_{n}\right\rangle_{\text {rel }}^{t}
$$

so that another prescription is needed to construct $\rho_{\text {rel }}(t)$, different from the consideration of closed systems in Section 4.

The relevant statistical operator. The principle of maximum information entropy at given mean values of a set of relevant observables $\left\{\mathrm{B}_{n}\right\}$ was used to construct $\rho_{\text {rel }}(t)$, Equation (44), in Section 4. We applied this condition also in the case of open systems where some parameter values $\lambda_{n \text {,ext }}$ corresponding to $\left\{\mathrm{B}_{n, \text { ext }}\right\}$ are prescribed by the external conditions. Other dynamical observables $\left\{\mathrm{B}_{n, \text { resp }}\right\}$ will show a response to the external conditions. Averages $\left\langle\mathrm{B}_{n, \text { resp }}\right\rangle^{t}$ of further observables are not measured. The self-consistency conditions to eliminate the corresponding response parameters $\lambda_{n, \text { resp }}(t)$ become unfounded. In Section 5 , the self-consistency conditions have been replaced by the condition of stationarity, where the time evolution of $\left\langle\mathrm{B}_{n}\right\rangle^{t}$ is determined only by $\mathrm{H}^{t}$. We propose another prescription. The response parameters $\lambda_{n, \text { resp }}(t)$ of a given set $\left\{B_{n, \text { resp }}\right\}$ of relevant observables are determined by the principle of maximum entropy production. As discussed in Section 5 , in the case of linear response, the corresponding Kohler variational principle is equivalent to the solution of the stationarity conditions with (43) and (93). The selection of a set $\left\{\mathrm{B}_{n, \text { resp }}\right\}$ of relevant observables is a variational ansatz to determine an optimal relevant statistical operator $\rho_{\text {rel }}(t)$ to which the system tends to evolve, as a response to the external influences. It is not dependent on the initial conditions and may be discussed in the context with the experimental evidence of universal dynamics far from equilibrium during the relaxation process observed recently [30].

Relaxation time. The source term to describe the influence of the bath on the time evolution of $\rho(t)$, Equation (83), contains the parameter $\eta$, which may be interpreted as inverse relaxation time and is, in general, a superoperator acting in the space of observables $\left\{B_{n}\right\}$ of the system. As an example, the electrical DC conductivity $\sigma\left(n_{e}, T\right)$ of the Lorentz model plasma was discussed. Different values $\eta_{\mathrm{e} i}, \eta_{\text {bath }}$ were considered for the relaxation of the electron current and the internal energy, respectively. The values were determined from the calculation of the corresponding microscopical processes, the $e-i$ interaction, and the bremsstrahlung emission, respectively. For comparison, in kinetic theory, a relaxation time tensor (21) was introduced, which depends on the wave number $p$. As known from a variational principle, a larger set $\left\{\hat{\mathrm{B}}_{n}\right\}$ of relevant observables will improve the result for the calculation of $\sigma$ using perturbation theory, so that the value of $\sigma$ increases; see Section 5 .

Stochastic processes. In Brownian motion, the friction term is connected with a stochastic process. As a famous relation, the friction coefficient is related to the fluctuation strength of the stochastic forces. Therefore, we expect that the von Neumann equation with the relaxation term has to be replaced by some stochastic process; see [4]. In the case of the relaxation of the electron current, we have considered the dynamical collision frequency, which is related to the correlation function of stochastic forces from the $e-i$ interaction. The fluctuations of the electromagnetic fields (vacuum, as well as thermal) are related to the transition rates for photon emission, in particular the bremsstrahlung emission describing the export of energy.

Energy flow. The incoming power density is given by the classical electromagnetic fields, in particular the Poynting vector, which couple to the electrons. We do not need any information about how these fields are produced. The export of energy, described by the relaxation term, is realized by the interaction with the bath. Details about the interaction with the bath are not of relevance. The electron-ion system acts via the force-force correlation function. It mediates and limits the flow of energy, but does not produce entropy. Mechanical energy is transferred to radiation to be described by quantum electrodynamics. Reabsorption transforms the radiation spectrum to the Planck distribution as thermal equilibrium with a fixed temperature $T_{\text {ext }}$. The corresponding excitation energy in addition to the vacuum part, the thermal part, may be denoted as heat. The limiting value $\omega \rightarrow 0$ of the spectral 
density of the radiation field, the Rayleigh-Jeans law $B(\omega, T)=\omega^{2} k_{\mathrm{B}} T / 2 \pi^{2} c^{2}$, can be used to define a local temperature.

Heat production and entropy. Electrical conductivity describes a non-equilibrium process. Directed motion that is obtained from the external field is converted into isotropic, undirected motion after the interaction with ions. This interaction is a reversible motion, so that it is not connected with the production of thermodynamic entropy $S_{\text {th }}$. Irreversibility is connected with the production of entropy. This means that in the case of electrical conductivity, heat is produced. An interesting process to transfer energy from the system to the bath is radiation, in particular the bremsstrahlung emission. The formation of a Planck spectrum can be identified as the production of heat. In equilibrium, heat cannot be transformed back to work, which means irreversible evolution.

Outlook. The results presented here are only a step toward a more fundamental approach to describe nonequilibrium processes. The stochastic properties of the electromagnetic fields should be analyzed more in detail to obtain a solution of the problem of irreversible evolution, including the electrical conductivity of a plasma. Using the Zubarev NSO method, exact results can be given for the DC conductivity in the low-density limit similar to a virial expansion, as discussed in the present work. This approach is extended to describe open systems, in particular the coupling to the radiation field.

Funding: This research received no external funding.

Acknowledgments: The author thanks V. G.Morozov, D. Blaschke, R. Redmer, and H. Reinholz for valuable comments and discussions. Work was initiated by the Symposium on "Nonequilibrium Phenomena in Strongly Correlated Systems" in Dubna, 16 April 2018.

Conflicts of Interest: The authors declare no conflict of interest.

\section{References}

1. Zubarev, D.N. Nonequilibrium Statistical Thermodynamics; Plenum Press: New York, NY, USA, 1974.

2. Zubarev, D.; Morozov, V.; Röpke, G. Statistical Mechanics of Nonequilibrium Processes; Akademie-Verlag: Berlin, Germany, 1996; Volume 1.

3. Zubarev, D.; Morozov, V.; Röpke, G. Statistical Mechanics of Nonequilibrium Processes; Akademie-Verlag: Berlin, Germany, 1997; Volume 2.

4. Röpke, G. Nonequilibrium Statistical Physics; Wiley-VCH: Weinheim, Germany, 2013.

5. Röpke, G. Nonequilibrium Statistical Operator. arXiv 2019, arXiv:1905.02012. [CrossRef]

6. Röpke, G. Electrical Conductivity of Charged Particle Systems and Zubarev's Nonequilibrium Statistical Operator Method. Theor. Math. Phys. 2018, 194, 74. [CrossRef]

7. Kramers, H. On the theory of X-ray absorption and of the continuous X-ray spectrum. Philos. Mag. 1923, 46, 836. [CrossRef]

8. Gaunt, J. Continuous absorption. Proc. R. Soc. Lond. A 1930, 126, 654. [CrossRef]

9. Boltzmann, L. Vorlesungen über Gastheorie, II Theil; Verlag J. A. Barth: Leipzig, Germany, 1898.

10. Bogoliubov, N.N. Problems of Dynamic Theory in Statistical Physics; Gostekhizdat: Moscow-Leningrad, Russia, 1946. (In Russian)

11. Röpke, G. Quantum-statistical approach to the electrical conductivity of dense, high-temperature plasmas. Phys. Rev. A 1988, 38, 3001. [CrossRef]

12. Röpke, G.; Redmer, R. Electrical conductivity of nondegenerate, fully ionized plasmas. Phys. Rev. A 1989, 39, 907. [CrossRef]

13. Redmer, R. Physical properties of dense, low-temperature plasmas. Phys. Rep. 1997, 282, 36. [CrossRef]

14. Reinholz, H. Dielectric and optical properties of dense plasmas. Ann. Phys. (Paris) 2005, 30, 1. [CrossRef]

15. Spitzer, J.L.; Härm, R. Transport Phenomena in a Completely Ionized Gas. Phys. Rev. 1953, 89, 977. [CrossRef]

16. Reinholz, H.; Röpke, G. Dielectric function beyond the random-phase approximation: Kinetic theory versus linear response theory. Phys. Rev. E 2012, 85, 036401. [CrossRef]

17. Gocke, C.; Röpke, G. Master equation of the reduced statistical operator of an atom in a plasma. Theor. Math. Phys. 2008, 154, 26. [CrossRef]

18. Lin, C.; Gocke, C.; Röpke, G.; Reinholz, H. Transition rates for a Rydberg atom surrounded by a plasma. Phys. Rev. A 2016, 93, 042711. [CrossRef] 
19. Christoph, V.; Gocke, C. Theory of Inverse Linear Response Coefficients. Phys. Status Solidi (b) 1985, $131,11$. [CrossRef]

20. Röpke, G. Dielectric function and electrical DC conductivity of nonideal plasmas. Phys. Rev. E 1998, $57,4673$. [CrossRef]

21. Kalashnikov, V.P. Linear relaxation equations in the nonequilibrium statistical operator method. Theor. Math. Phys. 1978, 34, 412. [CrossRef]

22. Röpke, G. Electrical conductivity of a system of localized and delocalized electrons. Theor. Math. Phys. 1981, 46, 184. [CrossRef]

23. Adams, J.R.; Shilkin, N.S.; Fortov, V.E.; Gryaznov, V.K.; Mintsev, V.B.; Redmer, R.; Reinholz, H.; Röpke, G. Coulomb contribution to the direct current electrical conductivity of dense partially ionized plasmas. Phys. Plasmas 2007, 14, 062303. [CrossRef]

24. Breuer, H.P.; Petruccione, F. The Theory of Open Quantum Systems; Oxford University Press: Oxford, UK, 2006.

25. Reinholz, H.; Redmer, R.; Röpke, G.; Wierling, A. Long-wavelength limit of the dynamical local-field factor and dynamical conductivity of a two-component plasma. Phys. Rev. E 2000, 62, 5648. [CrossRef]

26. Röpke, G.; Selchow, A.; Wierling, A.; Reinholz, H. Lindhard dielectric function in the relaxation-time approximation and generalized linear response theory. Phys. Lett. A 1999, 260, 365. [CrossRef]

27. Huba, J.D. 2013 NRL Plasma Formulary, 2013 Revision, p. 58. Available online: https:/ / library.psfc.mit.edu/ catalog/online_pubs/NRL_FORMULARY_13.pdf (accessed on 6 June 2019)

28. Ivanov, Y.V.; Mintsev, V.B.; Fortov, V.E.; Dremin, A.N. Electric conductivity of a non-ideal plasma. ZhETF 1976, 71, 216; English translation: JETP 1976, 44, 112.

29. Joos, E.; Zeh, H.D.; Kiefer, C.; Giulini, D.; Kupsch, J.; Stamatescu, I.O. Decoherence and the Appearance of a Classical World in Quantum Theory, 2nd ed.; Springer: Berlin/Heidelberg, Germany, 2003.

30. Erne, S.; Bücker, R.; Gasenzer, T.; Berges, J.; Schmiedmayer, J. Observation of universal dynamics in an isolated one-dimensional Bose gas far from equilibrium. Nature 2018, 253, 225. [CrossRef] [PubMed]

(C) 2019 by the authors. Licensee MDPI, Basel, Switzerland. This article is an open access article distributed under the terms and conditions of the Creative Commons Attribution (CC BY) license (http:/ / creativecommons.org/licenses/by/4.0/). 